\title{
Design of an Accurate and Stiff Wooden Industrial Robot: First Steps towards Robot Eco-sustainable Mechanical Design
}

\author{
Sébastien Briot* \\ Centre National de la Recherche \\ Scientifique (CNRS) \\ Laboratoire des Sciences du Numérique \\ de Nantes (LS2N), UMR CNRS 6004 \\ 44321 Nantes, France \\ Email: Sebastien.Briot@Is2n.fr
}

\author{
Clément Boudaud \\ LIMBHA \\ Groupe École Supérieure du Bois \\ 44306 Nantes, France \\ Email: clement.boudaud@ecoledubois.fr
}

\author{
Pauline Lafoux \\ École Centrale de Nantes \\ Laboratoire des Sciences du Numérique \\ de Nantes (LS2N), UMR CNRS 6004 \\ 44321 Nantes, France \\ Email: paulinelafoux@gmail.com
}

\author{
Lila Kaci \\ École Centrale de Nantes \\ Laboratoire des Sciences du Numérique \\ de Nantes (LS2N), UMR CNRS 6004 \\ 44321 Nantes, France \\ Email: lilakaci91@yahoo.fr
}

David Llevat Pamiès

École Centrale de Nantes

Laboratoire des Sciences du Numérique

de Nantes (LS2N), UMR CNRS 6004

44321 Nantes, France

Email: david.llevat@gmail.com

\author{
Philippe Martinet \\ Université Côte d'Azur, Inria \\ 06902 Sophia Antipolis, France \\ Email: Philippe.Martinet@inria.fr
}

This paper investigates the feasibility of replacing metal robot links by wooden bodies for eco-sustainable design's purpose. Wood is a material with low environmental impact, and a good mass-to-stiffness ratio. However, it has significant dimensional and mechanical variabilities. This is an issue for industrial robots that must be accurate and stiff.

To guarantee stiffness and accuracy performance of a wooden robot, we propose an integrated design process combining (i) proper wood selection, (ii) adequate sensor-based control strategies to ensure robot accuracy and (iii) a robust design approach dealing with wood uncertainties.

Based on the use of this integrated design process, a prototype of a wooden five-bar mechanism is designed and manufactured. Experimental results show that it is realistic to design a wooden robot with performance compatible with Industry requirements in terms of stiffness ( deformations lower than 400 microns for $20 \mathrm{~N}$ loads) and accuracy (repeatability lower than 60 microns), guaranteed in a workspace of $800 \mathrm{~mm} \times 200 \mathrm{~mm}$. These works provide a first step towards the eco-sustainable mechanical design of robots.

\footnotetext{
*Address all correspondence to this author.
}

\section{Introduction}

In order to meet expectations of the New Industrial Revolution [1], Robotics faces ten grand challenges [2] from the use of new materials and fabrication schemes, to robot ethics, passing through bio-hybridation or exploration. A transversal challenge is robot eco-sustainability, for supporting Climate Change Mitigation and limiting the impacts of human activities. As a matter of fact, eco-sustainability was identified as a world Challenge. Therefore, eco-sustainability is a key issue of future robot development.

Until recently, works on robot eco-sustainability were limited to energy saving, because this issue has a strong financial impact for Industry [3]. Many results on mechanical design of lightweight or balanced robots [4,5], optimal trajectory planning [6,7] energy harvesting and allocation [8, 9], or also new actuation strategies [10], have been obtained.

However, considering robot eco-sustainability solely from the viewpoint of energy consumption fails to address major environmental impacts (defined in the norms ISO 14040 [11] and ISO 14044 [12]) related to its design: a large part of robot environmental impact is due to the use of materials like metals, synthetic polymers or carbon composites 
for the manufacturing of its mechanical components [13].

Addressing robot eco-sustainability from the perspective of the robot mechanical architecture design is new and very little explored. Material recycling is a first step towards this perspective, and is already envisaged by some robot manufacturers like Yaskawa. However, recycling processes have non-negligible environmental impacts, or even, do not exist for carbon composites used in lightweight robots [14]. To overcome recycling issues, some researchers use new materials in a robot design context: bio-sourced materials. Bio-sourced materials have a very low environmental impact [14], are renewable and their availability is only limited by their growing time. The net emissions of carbon per ton $(\mathrm{C} / \mathrm{t})$ of produced material are $-457 \mathrm{~kg} \mathrm{C} / \mathrm{t}$ for structural wood, $694 \mathrm{~kg} \mathrm{C} / \mathrm{t}$ for steel and $4532 \mathrm{~kg} \mathrm{C} / \mathrm{t}$ for aluminium according to [15], even considering that comparison should be based on broader functional unit, orders of magnitude show an intrinsic interest for bio-sourced materials. Also, rigidity-to-density ratios are relatively similar. With modulus of rigidity (longitudinal for the wood) of $210 \mathrm{GPa}$, $69 \mathrm{GPa}$ and $11 \mathrm{GPa}$ for steel, aluminium and wood (mean value) [16] and respective densities of 7.8, 2.7 and 0.42 (mean value for wood), the ratios are respectively of 26.9, 25.5 and 26.2. Moreover, most of these bio-sourced materials are bio-degradable, thus making them attractive for use in ecologically fragile environments.

Bio-sourced materials have been already tested in different robotics contexts. For small sized robots, Bioglycerine [17] or muscle fibres [18] have been employed to design small-size actuators. Bio-degradable transistors have been developed in [19]. The use of plants was also investigated in [20] for creating bio-hybrid robots for the definition of architectural ambiances. Bigger robots represent most of the robot stock and, as a result, have a much more considerable global environmental impact. First attempts of using wood [21, 22], bamboo [23] or even bio-composites [24, 25] for designing industrial robots have been achieved. These plant-based bio-sourced materials have good stiffness-tomass ratio, and are thus appealing for eco-designing bigger size robots [26]. However, they all have the same issues: their performance and dimensions vary with the atmospheric conditions or external solicitations and with the conditions in which they were grown [26, 27, 28]; How to be sure that a robot made with these materials will have a performance compatible with its use in Industry, like being accurate, stiff, and having few vibrations, even if plant-based bio-sourced materials properties vary? In this work, we provide answers to several of these questions.

In [21, 22], the authors replaced proximal links of a Delta-like robot made in carbon composites by wooden arms, for eco-design purpose. They made several prototypes of arms with different shapes and different species of wood, and took the one with the best ratio of mass (or inertia) to deformation. In [23], the authors designed a robot in bamboo by replacing the tubular bodies of the Schunk modular robot arm by bamboo tubes. A computation of the robot stiffness was made in order to have an estimation of its deformation under loading. In [24 25], the authors optimized the dimension of a beam with an elliptic cross-section made of bio-composites in order to replace a body of a six-degrees-of-freedom parallel robot prototype. They modeled the composite behavior and showed that a robot made of bio-composite may have better behaviors in terms of damping and amplitudes of vibrations than a metal robot.

However, in all these works, the problem of the variation of the mechanical properties was not investigated at all. All authors checked in simulation, and sometimes experimentally, the robot deformations under loading for a mean value of the Young's modulus of the material under consideration. Their models can be thus used for a single prototype. However because of the variability of the material elasticity moduli in the robot links, these models cannot be used in the design process in order to give anticipated predictions of the robot deformations. This is a problem, because for standard wood species (like pine), the Young's modulus value of two different wooden beams may vary up to $\pm 30 \%$ with respect to a nominal value [26]. Therefore, it is impossible to predict in advance the variability of the elastic performance of the robot, and thus it is difficult to have confidence in the reliability of the robot design in terms of stiffness performance.

Additionally, none of the works [21, 22, 23, 24,25] deals with the problem of the dimensional variability of the robot made of plant-based bio-sourced materials under the variation of the atmospheric conditions. In [29], the authors proposed an external measurement machine that is able to improve the accuracy of a 3-degrees-of-freedom (DOF) parallel robot. In their conclusion, they claimed that this exteroceptive mechanical system could be used in order to ensure the accuracy performance of a robot whose parts could be made of wood. However, no simulations or experimentations are provided in order to support their claim.

The main contributions of this paper are of several types:

- Conceptual contribution: we propose a new framework (an integrated robot design approach) that is able to deal with the design of a wooden industrial robot, for ecodesign purposes, with guaranteed performance in terms of accuracy and stiffness.

- Theoretical contributions: The proposed integrated design approach requires revisiting the way the optimization algorithms for robot design are structured. Therefore, we propose new formulations for the design algorithms which are the scientific novelties of this paper,

- On control-based design: We develop an optimization procedure for taking into account the sensor-based controller performance.

- On reliable topology optimization: We modify an existing approach [30] for topology optimization and adapt it to our class of problems. Modifications between [30] and our work are not minor: (a) we define a proper reliable design criterion that was not provided in the work [30] (b) we guarantee the performance in the full workspace, which is not the case in [30]; (c) finally, we provide simulation results in order to show that the reliable design approach is valid, i.e. it leads to a limited 
number of designs that would not ensure the deformation performance of the robot.

- Experimental contribution: We perform a significant number of experiments in order to characterize the robot performance in terms of accuracy and deformation, in order to show that our theory is not just valid in theory, but also in practice.

The paper is an extended version of [31] where we provide results on the deformation under loading of a wooden industrial robot prototype, and we develop several sensorbased control approaches and experimentally compare their performance in terms of robot accuracy, A first discussion about the obtained decrease of the environmental impact when using wood for industrial robot design was provided.

The paper is thus divided as follows. In Section 2, the advantages and drawback of the wood in the context of robot eco-sustainable mechanical design, and the robot design requirements are specified. A description of the integrated design approach is given. In Section 3, we start by defining the controllers we decided to test for ensuring the accuracy performance of the wooden robot. Then we formulate the design optimization problem used in order to optimize the primary geometric parameters of the robot, i.e. its link lengths [32]. In Section 4, a reliable topology optimization approach able to guarantee the maximal deformation of the robot even if the material elasticity properties are uncertain is proposed in order to define the optimal robot link shapes. Section 5 describes the wooden robot prototype and provides experimental results for the robot deformation and accuracy. In Section 6 a discussion about the experiments is given. Finally, in Section 7, conclusions are drawn.

\section{Description of the wooden robot design approach}

In this Section, we first propose an integrated design methodology able to ensure that a wooden robot can be stiff and accurate. Then, we detail the industrial robot design specification and chosen kinematic architecture.

\subsection{Advantages and drawbacks of wood in the context of robot eco-sustainable mechanical design}

Wood is one of the many bio-sourced materials that could help reducing the environmental impact of robots. It was chosen in the context of this work for several reasons: (a) its main advantages mentioned in the introduction (stiffness to density ratio equivalent to steel or aluminum, low environmental impact); (b) it is relatively well known compared to other newer materials (e.g. natural fibre composites); (c) its availability and ease of use in this project. As previously mentioned, wood presents some challenges as an engineered material: (i) there is a large variety of species, leading to questions about the best ones to be used for robot design, (ii) wood expects a variability of its properties, has some issues of dimensional stability, long-term behaviour (creep), etc.

The issue of the variability of mechanical properties is predominant, as it directly affects the elastic (instantaneous) behaviour of the robot under loading, leading to difficulties in predicting its deformations. Dimensional stability depends on the evolution of hygrothermal conditions. The choice of a very specific type of wood (e.g. acetylated beech and pine described in the next Section) may help reducing the impact of this problem for robot accuracy. Moreover, reducing the impact of the wood dimensional stability makes it possible to give more focus on the already complex experimental work for characterizing the elastic behaviour of the robot.

\subsection{Proposed design methodology}

As mentioned above, at least two main performances must be achieved by a wooden industrial robot: accuracy and stiffness. However, The dimensions of bodies made of wood vary with their moisture content (a function of temperature and relative humidity) [27], which raises issues in terms of robot accuracy. Moreover, The wood mechanical characteristics vary greatly even between two pieces of wood of the same species [26], which raises issues for guaranteeing the robot stiffness.

With respect to the issues in terms of robot accuracy, we made the hypothesis that it was possible to solve them thanks to a combination of:

1. A proper choice of wood: acetylated wood, i.e. wood which has been treated with acetic anhydride in order to increase its dimensional stability and durability with very little change on its mechanical behaviour [27]. Moreover, acetylation is a non-toxic chemical treatment (which produces acetic acid has a by-product). Proportionaly the acetylation process has a significant effect on the environmental impact of acetylated wood (compared to untreated wood) [33], but the gain in dimensional stability, durability and its still very low level of $\mathrm{kgCO}_{2 \mathrm{eq}}$ per $\mathrm{m}^{3}$ compared to others materials [34], makes it a good alternative for commonly used materials in robotics. Two species of wood were studied: acetylated pine (Pinus Radiata, softwood) and acetylated beech (Fagus Sylvatica, hardwood).

In the following of this work, we decided to use acetylated beech for designing the robot, because beech is stiffer than pine, even if it presents larger dimensional variability [26].

2. The use of proper control approaches: by using sensorbased controller, an accurate control of the robot positioning can be obtained [35,36] in order to compensate the error due to the dimensional variability of the wooden bodies and to ensure the desired accuracy. Indeed, these types of controllers, which gives the ability for the robot to react in any change in its environment, and more generally, to any evolution of the interaction between the robot and the environment, are more and more used in industrial context. However, it was shown in [37] that these types of controllers can meet singularities near which accuracy performance is considerably decreased, and these types of singularities may depend both on controller parameters and robot geometry (essentially the relative distances and orientations between 
the robot joints, i.e. the robot primary geometric parameters [32]. Therefore, the optimal design methodology must define the optimal robot primary geometric parameters such that:

(a) It is possible to ensure that there are not any controller singularities inside the robot workspace,

(b) A relevant accuracy model is used: accuracy performance must be computed by taking into account that external sensors used in the controller will ensure the accuracy, instead of the standard motor encoders.

In other words, it is necessary to develop a controlbased design methodology.

With respect to the issues in terms of robot stiffness, we believe that this problem can be tackled through an optimal design of link shapes. Indeed, as it was shown in [30], it is possible to take into account the probability distribution of the Young's modulus in order to compute the expectation and standard deviation of the link deformation under given loading. It is feasible to adapt this work for computing robot deformations and their variability, and to perform a reliable robot topology optimization.

In order to summarize, thanks to an integrated design approach based on:

1. a proper choice of wood type,

2. the use of adequate sensor-based controllers,

3. the definition of a proper design methodology, taking into account the controller performance and the variability of the elastic characteristics of the wood,

we believe that it is possible to design an industrial wooden robot with performance in terms of accuracy and stiffness that can be guaranteed.

We should also mention that, because the controller accuracy performance depends essentially on the robot primary geometric parameters, while the stiffness performance depends on both the links lengths and shapes, the optimal design problem can be solved in cascade, as it was done in [38]: a first optimization problem will define the optimal primary geometric parameters and a second optimization problem will find the optimal link shapes.

The next section deals with the specifications which have been chosen for the design of our wooden robot.

\subsection{Design specifications}

The performance to be achieved by the wooden industrial robot has been fixed by our partners in the scope of the project RobEcolo [31]. They are detailed in Tab. 1. The robot must be a two-DOF translational robot moving in the horizontal plane, with a regular dextrous workspace (i.e. a workspace in which all desired performance are satisfied [39]) which is a rectangle of dimension $800 \mathrm{~mm} \times$ $200 \mathrm{~mm}$. The performances to be satisfied are the repeatability and deformations in the plane of the motion under given loadings, which are detailed in Tab. 1. The objective (not given in the Table) is that the robot must be as compact as possible, as it was done in [38].

The decision to design a two-DOF translational robot is motivated by the facts that:

- Funding constraints of the project RobEcolo which limited to possibility to design a robot with a lot of degrees of freedoms

- Many industrial tasks are limited to work on a plane or in a cylinder. As a result, many industrial robots are two-DOF planar robots or four-DOF robots for SCARA motions. They are particularly used in electronics Industry, which represents a major sector for robots use and sells [40]. This last type of robots is pretty often made of a planar two-DOF manipulator which positions a tool which achieved vertical translations and rotations.

As a result, the partners of the project RobEcolo decided to focus on these types of robot manipulators.

With respect to the robot mobility requirements, we decided to design a five-bar mechanism [41] (Fig. 1). Designing a parallel robot instead of a serial one was decided because of the known design complexity of parallel robots. Indeed, their physical performances are much more affected by the change of their configurations than performances of serial robots, especially near parallel singularities [39 $\left[\begin{array}{l}1 \\ \text { Thus, }\end{array}\right.$ being able to prove on a parallel robot that our methodology is able to ensure good performance is a relevant index that the methodology could work on a less complex architecture.

It is a two DOF planar parallel robot with two translations in the plane $\left(\boldsymbol{x}_{0} O \boldsymbol{y}_{0}\right)$. The end-effector is positioned at point $C$. The mechanism is made of four links: (i) two proximal links which are the bodies between $A_{i}$ and $B_{i}(i=1,2)$ and (ii) two distal links which are the bodies between points $B_{i}$ and $C$. The proximal and distal links are connected by three passive revolute joints while the joints located at point $A_{1}$ and $A_{2}$ are active.

Based on the fact that it has been decided to design a parallel robot, an additional design constraint is to have no singularity in the dextrous workspace. Moreover, as explained later, a camera will be used in order to observe either the robot end-effector or distal links. Thus, in order to facilitate the observation of these distal links, they are imposed to be of cylindrical shapes.

It should be mentioned that, with respect to the challenge of designing an industrial wooden robot with guaranteed performance, which has never been done before, some simplifications of the design problem have been decided by the RobEcolo project partners:

- No constraints on the deformations along $\mathbf{z}_{0}$ have been specified, because we decided to focus on the design of a robot which is accurate in its plane of motion. However,

\footnotetext{
${ }^{1}$ While the physical performances of parallel robots are much more affected by the change of their configurations, in comparison with serial robots, this is not the case for the controller performance: Both parallel and serial robots can be controlled using the same standard strategies: PID or Computed torques control [42 43].
} 


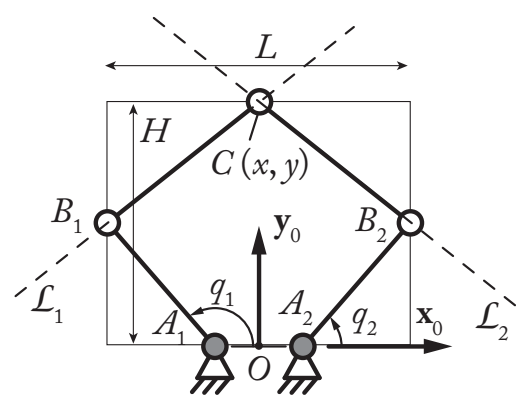

Fig. 1. Kinematic scheme of a five-bar mechanism

these vertical deformations will be checked a posteriori in the design process: it will be verified on CAD and on the real prototype that they are "acceptable", which means for us, around 500 microns under a loading of $20 \mathrm{~N}$ along $z_{0}$;

- The robot is a prototype made for validating performance in terms of stiffness and accuracy; therefore no dynamic properties have been imposed. However, the design process that will be proposed should tend to reduce the mass or robot link inertia so that it avoids leading to the manufacture of bulky links.

Finally, the number and types of sensor used in order to ensure the robot positioning accuracy have been imposed by the project partners. With respect to the desired $500 \mathrm{mi}-$ crons of absolute positioning accuracy specified in Tab. 1. we propose to use a single camera, the Toshiba $\mathrm{Bu} 238 \mathrm{M}$ $(1920 \times 1200$ pixels of resolution and a focal length of $8 \mathrm{~mm}$ as sensors (in order to limit the distortion) and a grand angular objective, specifically, with a field of view of around $80 \mathrm{deg} \times 60 \mathrm{deg}$ ).

As proposed in the Subsection 2.2, the design optimization problem will be solved in cascade, starting from the optimization of the primary geometric parameters. As a result, next Section introduces the formulation of the design optimization problem used in order to optimize the fivebar mechanism primary geometric parameters, i.e. its link lengths.

\section{First optimization problem: Finding the optimal link lengths of the five-bar mechanism}

In this Section, we are going to formulate and solve the optimization problem leading to the definition of the optimal link lengths of the five-bar mechanism that will ensure its accuracy. As previously mentioned, in order to compensate the errors due to the dimensional variability of the wooden bodies and to ensure the desired accuracy of the robot, visual servoing will be used. However, these types of controllers can meet singularities near which accuracy performance is considerably decreased, singularities which depend both on controller parameters and robot link length. Therefore, before all, it is necessary to define which types of controllers will be used for tackling with the positioning accuracy issues.

\subsection{Choice of the vision-based controllers}

Visual servoing is a control technique which uses feedback information extracted from a vision sensor (visual feedback) to control the motion of a robot [44]. Many different vision-based controllers exist, e.g. observation of points [35], lines [45], or image moments [46]. Given some measurements $\mathbf{s}$ extracted from a camera (or a set of cameras), it is possible to define an error of observation $\mathbf{e}=\mathbf{s}-\mathbf{s}^{*}$, where $\mathbf{s}^{*}$ is the desired visual feature to be attained. Knowing the kinematic interaction model between the camera and the object to be observed which takes the generic form:

$$
\dot{\mathbf{s}}=\mathbf{L}(\mathbf{s}, \boldsymbol{\pi}) \mathbb{V}
$$

where $\mathbb{V}$ is the relative twist between the object and camera frames and $\mathbf{L}(\mathbf{s}, \boldsymbol{\pi})$ is the interaction matrix which depends on the measurements $\mathbf{s}$ (and that is not necessarily square) and, for some controllers, on robot primary geometric parameters denoted as $\boldsymbol{\pi}$ (for the five-bar mechanism, they are the link lengths and the diameter of the observed cylindrical links [47]). Then, it comes pretty easily that:

$$
\dot{\mathbf{e}}=\mathbf{L}(\mathbf{s}, \boldsymbol{\pi}) \bigvee
$$

In the following of this work, a camera will be mounted on the robot fixed frame (eye-to-hand control strategy [35]); thus $\mathbb{V}$ represents the robot end-effector twist. Imposing an exponential decoupled decrease of the error (i.e., $\dot{\mathbf{e}}=-\lambda \mathbf{e}$ with $\lambda$ a positive real number), we obtain using (2):

$$
\mathbb{V}=-\lambda \mathbf{L}^{+}(\mathbf{s}, \boldsymbol{\pi}) \mathbf{e}
$$

in which $\mathbf{L}^{+}(\mathbf{s}, \boldsymbol{\pi})$ is the pseudo-inverse of $\mathbf{L}(\mathbf{s}, \boldsymbol{\pi})$. Passing from the Cartesian space control to the joint space control can be done thanks to the use of the robot kinematic Jacobian matrix $\mathbf{J}(\mathbf{q})$ linking the twist $\mathbb{V}$ to the robot joint velocities $\dot{\mathbf{q}}$ by the relationship: $\mathbb{V}=\mathbf{J}(\mathbf{q}) \dot{\mathbf{q}}$, as is done in Section 5 . More details on visual servoing can be found in [35, 44, 48].

To the best of our knowledge, only few types of visual servoing techniques have been applied to the control of parallel robots: for instance direct observation of the end-effector pose [49] (case A, Fig. 2(a)], or observation of the robot leg projections in the image (Fig. 2(b) and reconstruction of their directing lines [47] (case B) or directions [36] (case C).

We decided to test controllers of cases A and B because the observation of the end-effector is by essence more suitable in order to ensure the end-effector positioning accuracy (there is a direct observation of the object we want to control (no necessity to use unperfect models with unmodeled phenomena in order to control the robot)).

Moreover, when observing the position of the endeffector of the five-bar mechanism (represented as a point, Fig. 2(a)], the interaction matrix is square, diagonal and free of singularities [35]. However, depending on the endeffector's design, a target point on it might be difficult to observe. 
Table 1. Performance required for the wooden industrial robot

\begin{tabular}{|c|c|}
\hline Number and types of DOF & 2 translations in the horizontal plane $\left(\boldsymbol{x}_{0} O \boldsymbol{y}_{0}\right)$ \\
\hline Regular workspace size & $800 \mathrm{~mm} \times 200 \mathrm{~mm}$ \\
\hline Repeatability & 500 microns maximum in the regular workspace \\
\hline $\begin{array}{l}\text { End-effector deformations in the plane }\left(\mathbf{x}_{0} O \mathbf{y}_{0}\right) \text { under load- } \\
\text { ings } \mathbf{f}_{1}=\left[F_{x}=20 \mathrm{~N} F_{y}=0 \mathrm{~N} M_{z}=0 \mathrm{Nm}\right]^{T} \text { and } \mathbf{f}_{2}=\left[F_{x}=\right. \\
\left.0 \mathrm{~N} F_{y}=20 \mathrm{~N} M_{z}=0 \mathrm{Nm}\right]^{T}\end{array}$ & $\begin{array}{l}\left.u_{\max }=0.2 \mathrm{~mm} \text { in translation in the plane }\left(\boldsymbol{x}_{0} O \boldsymbol{y}_{0}\right)\right) \text { maximum in } \\
\text { the regular workspace }\end{array}$ \\
\hline
\end{tabular}

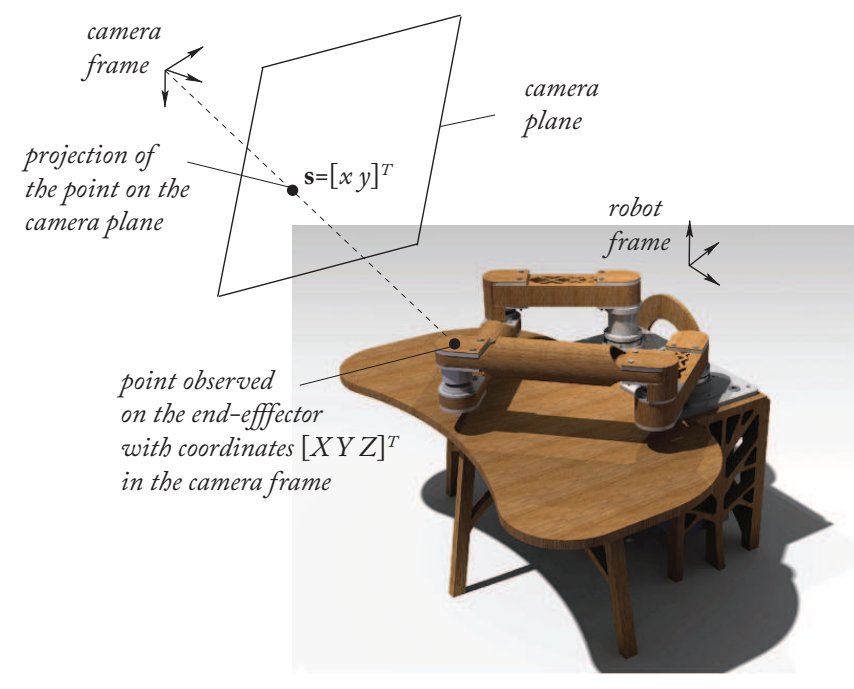

(a) Observation of the end-effector of the five-bar mechanism

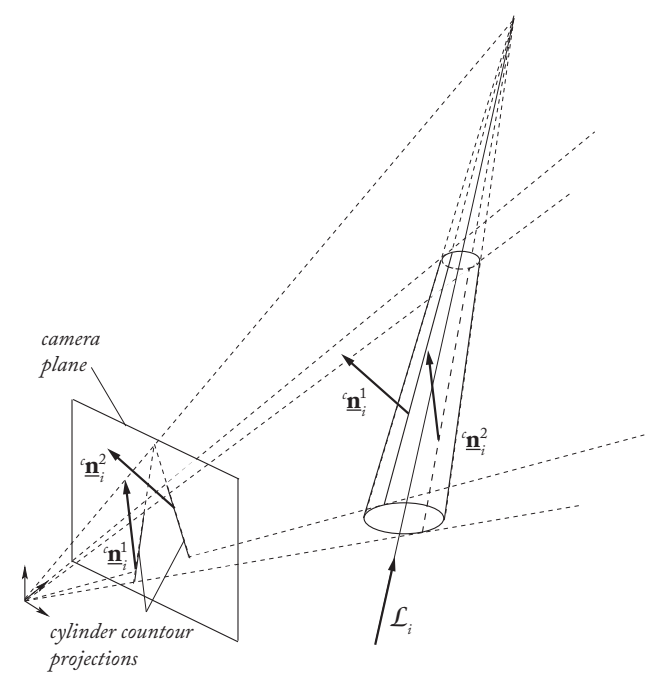

(b) Observation of a cylindrical leg of the five-bar mechanism

Fig. 2. Observation of different bodies for the five-bar mechanism.

Therefore, in case the controller of case A is not able to reach the accuracy performance, the controller of case B can be tested: as we observe the robot legs and not its endeffector, the interaction model takes the general form (1), i.e. it depends on the robot geometric parameters. This means that the interaction matrix is more sensitive to modeling issues such as joint clearance or leg dimensional variability (its interaction matrix depends on the camera location and also of the robot geometry), but legs are bigger and easier to observe than a single point [36]. Moreover, when applied to the control of the five-bar mechanism, it is known that the singularities of its interaction matrix are superposed with the Type 2 singularities of the five-bar mechanism [47], which is more convenient for robot mechanical design purpose: this may lead to the design of robots that will be more compact than if their workspace would have been compressed between two different types of singularities: those of the mechanical architecture, and those of the controller.

Controller of case $\mathrm{C}$ will be disregarded because its singularities appear in the middle of the workspace [47], thus encountering the drawback mentioned just previously.

\subsection{Characterizing the accuracy of the visual servoing}

Because a vision-based controller will be implemented in order to guarantee the robot accuracy, the end-effector position error $\delta \mathbf{p}$ will be due to the camera observation error $\delta$ s only, no more from the robot's encoder resolution (as it is the case in classic encoder-based control schemes [42]). This noise has been modeled by a random shift in the pixels:

- Case A: of the observed image point (Fig.2(a)

- Case B: of the pixel located where the image projection of the links edges meet the frame boundary (Fig. 3).

In our design process, a shift of \pm 0.1 pixel was considered: it is a standard pixellic error when using subpixellic image processing [50]. The observation error being small, we use the following error model based on the definition of the interaction model (1):

$$
\delta \mathbf{p}=\mathbf{L}^{+} \delta \mathbf{s}
$$

Even if this error model cannot characterize all error sources especially for the second controller (e.g. joint clearance, link deformation or manufacturing errors), it is computationally efficient and the final experimental results are compatible with the positioning accuracy computed using it. Thus, it will be used in order to characterize the robot accuracy during the design optimization process.

As explained in the paragraphs above, the interaction matrix of the first controller depends on the camera location only: thus its error model (4) depends on the camera location, and of the observation error. This is not the case for the second controller, which depends also of the robot geometry 


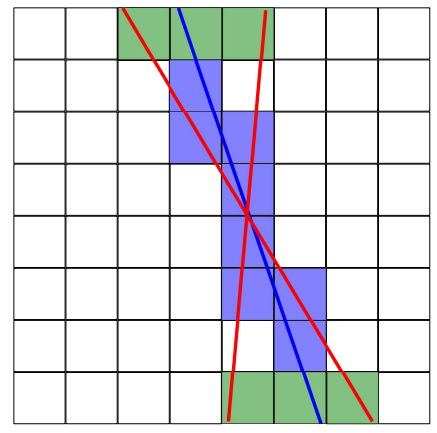

Fig. 3. Result of a one-pixel error on the intersection of the image boundary and the observed line: the line to be observed is in blue, its pixel appearance is in purple, and the potential reconstruction errors are the lines in red.

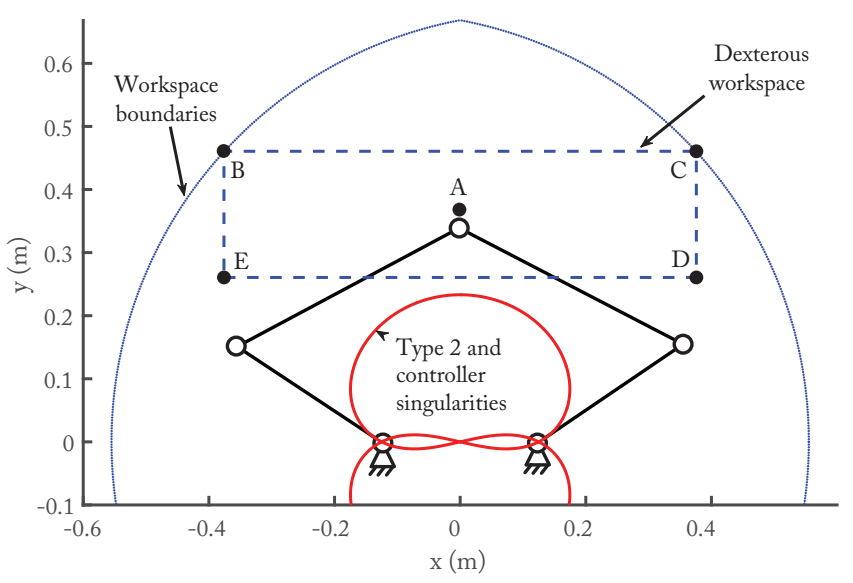

Fig. 4. Largest regular dexterous workspace of the optimized fivebar mechanism

$\pi$. As a result, in order to have small positioning errors for the second controller, it is necessary to find the robot link lengths leading to the desired accuracy. This is the topic of the next Subsection. It should be mentioned here that a single prototype will be made for both controllers because of the price of manufacturing process. Therefore, we optimized the robot geometry to have good performance for the second controller, and, because the first controller is not sensitive to robot geometry, it can be implemented on any types of 2DOF planar robots and, consequently, on the robot optimized for the second controller.

\subsection{Finding the optimal robot link lengths: Problem formulation and results}

Problem formulation: The optimization problem for defining the robot's links lengths and the camera location is formulated as follow. Based on the robot specifications given in Section 2.3, the objective is to minimize the footprint of the five-bar mechanism: we characterize the footprint as the surface covered by the robot in the configuration when its links $A_{i} B_{i}$ and $B_{i} C$ are orthogonal (for $i=1,2$, Fig. 1 1 . The constraints that we decide to take into account are that the regular dextrous workspace of the robot must be a rectan- gle whose dimensions should be bigger than $\ell_{W_{x 0}}=800 \mathrm{~mm}$ along $\mathbf{x}_{0}$ and $\ell_{W_{y 0}}=200 \mathrm{~mm}$ along $\mathbf{y}_{0}$. In this workspace, all following constraints must be satisfied:

- C1: the workspace must be free of Type 2 singularities [51],

- C2: the workspace must be free of singularities of the controller of case B: as mentioned earlier, in the case of the observation of the robot legs, these singularities are superposed with the Type 2 singularities,

- C3: the end-effector and the robot legs must be observable by the camera, meaning that in the projections of the observed objects must be contained in the camera image plane $(1920 \times 1200$ pixels of resolution, as mentioned earlier),

- C4: knowing the observation error of the camera $( \pm 0.1$ $\mathrm{px})$, the error on the end-effector position should be lower than 500 microns. The end-effector resolution is computed based on the error model given by Eq. (4) for the controller of case B,

- C5: the static forces exerted into the passive joints are proportional to $1 / \sin \xi, \xi$ being the angle between the distal links [38], it is decided consequently that $\sin \xi$ should be higher than 0.1 to avoid excessive efforts in the joints.

Thus, we formulated the following optimization problem:

$$
\begin{aligned}
\operatorname{minimize} & A=L H \\
\text { over } & \mathbf{x} \\
\text { subject to } & \ell_{W_{x}}>\ell_{W_{x 0}} \text { and } \ell_{W_{y}}>\ell_{W_{y 0}}
\end{aligned}
$$

where: $\mathbf{x}=\left[\begin{array}{llll}\ell_{0} & \ell_{1} & \ell_{2} & \mathbf{x}_{c}^{T}\end{array}\right]^{T}$ with $\ell_{0}=\ell_{A_{1} A_{2}}, \ell_{1}=\ell_{A_{1} B_{1}}=\ell_{A_{2} B_{2}}$ and $\ell_{2}=\ell_{B_{1} C}=\ell_{B_{2} C}$ are the robot link lengths, and $\mathbf{x}_{c}=$ $\left[x_{c} y c z c \phi \theta \psi\right]^{T}$ is a vector defining the position and orientation of the camera: $x_{c}, y_{c}$ and $z_{c}$ defining the position of the camera frame with respect to the world frame, $\phi, \theta$ and $\psi$ are the ZXZ Euler angles characterizing its orientation. $A=L H$ is the robot footprint (Fig. 11. $\ell_{W_{x}}$ and $\ell_{W_{y}}$ are the dimensions along $\boldsymbol{x}_{0}$ and $\boldsymbol{y}_{0}$ of the rectangular dextrous workspace in which all constraints 1 to 5 defined above must be satisfied [39]. The computation of $\ell_{W_{x}}$ and $\ell_{W_{y}}$ is based on the Algorithm presented in [32,38]. Then we look for the largest sub-matrix inside $\left\{\Omega_{i j}\right\}$ containing non-zero values only. The algorithm uses an additional integer matrix $\left\{\Phi_{i j}\right\}$ that defines the size of the candidate solutions workspace with the vertex $G_{i j}$. The validity of this routine and correctness of the relevant recurrent expression can be proven using the standard ideas of dynamic programming, similar to finding the largest square block in a two-dimensional binary matrix.

Before presenting the results obtained when running the optimization algorithm (5), it should finally be mentioned that the error model (4) for the controller of case B also depends on the shape and cross-section dimensions of the distal links [47] which were not included in our optimization problem in order to accelerate the computation of the results. As it was explained in Subsection 2.3. these links 
Table 2. Optimal link lengths for the wooden five-bar mechanism

\begin{tabular}{l|lll}
\hline \hline$A\left[\mathrm{~m}^{2}\right]$ & $l_{0}[\mathrm{~m}]$ & $l_{1}[\mathrm{~m}]$ & $l_{2}[\mathrm{~m}]$ \\
\hline 0.1372 & 0.125 & 0.280 & 0.400 \\
\hline \hline
\end{tabular}

Table 3. Optimal location of the camera

\begin{tabular}{llllll}
\hline \hline$x_{c}[\mathrm{~m}]$ & $y_{c}[\mathrm{~m}]$ & $z_{c}[\mathrm{~m}]$ & $\phi[\mathrm{rad}]$ & $\theta[\mathrm{rad}]$ & $\psi[\mathrm{rad}]$ \\
\hline 0 & 0.38 & 0.9 & 0 & $\pi$ & 0 \\
\hline \hline
\end{tabular}

are of cylindrical shapes, and we fix their diameter at $80 \mathrm{~mm}$.

Results of optimization: The optimal robot dimension and camera location are given in Tab. 2 and Tab. 3 respectively. The position of the regular dexterous workspace is shown in Fig. 4. We can clearly observe that it is free of the singularities of the robot (and also of the controller, as they are superposed for the chosen controller based on leg observation, as shown in [47]). The robot dimensions given in Tab. 2 will be the inputs of the next optimal design problem.

\section{Second optimization problem: Finding the optimal link shapes of the five-bar mechanism}

The link shape optimization is the most computational intensive step of the optimal robot design process. This optimization problem is based on the use of complex models for the computation of the robot elastic behavior, which must be computed thousands of times (and even more) in order to estimate the robot elastic performance in its whole workspace for a given set of design parameters [38]. This is achieved in order to verify that the performance can be guaranteed in the whole robot operational workspace [52]. Consequently, in order to decrease the computational expense, a common approach is to perform a parametric optimization [38] 53], i.e. reducing the number of design variables by modeling links using Bernoulli beam theory [54] and by considering that the shapes of their cross-sections is fixed (for instance, circle, square, rectangle, I-shape) and parameterized by a limited set of variables (e.g. radius for circles, edge lengths for rectangles, I-shapes). This approach is not the more accurate for finding the optimal link shapes, contrary to topology optimization [55]. Topology optimization requires more computational efforts and was not used in the past in robot design because it was thought not to be compatible with obtaining results in a reasonable amount of time. However, recent works [56, 57, 58 59] showed that this approach can be adapted for the purpose of robot design. Therefore, we are going to use it in the following of this Section.

Thus, we first briefly introduce the finite element models that have been used in the optimization process. Then, in order to deal with the problem of the wood mechanical performance variability, we propose a relevant performance index. Finally, we formulate the optimization problem and we solve it.

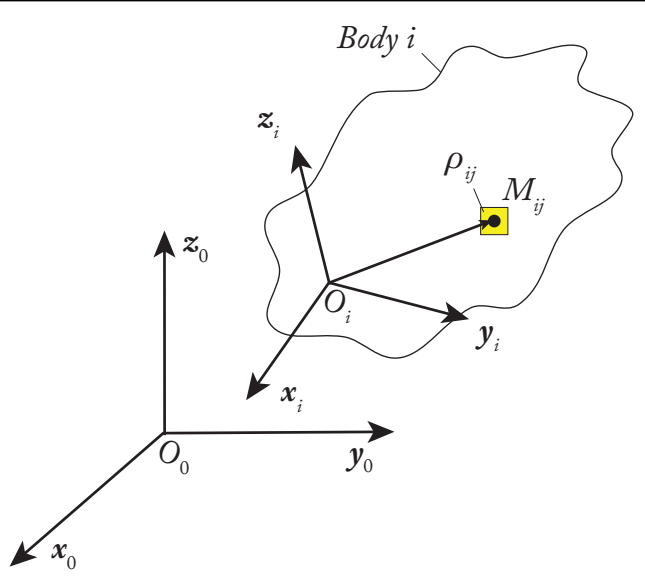

Fig. 5. A body in space meshed with finite elements.

\subsection{Modeling of the link elastic behavior}

In what follows, we consider the FEA of the five-bar mechanism, i.e. a robot with four moving bodies (Fig. 1). The body $i(i=1, \ldots, 4)$ is meshed with $m_{i}$ elements. The element $j$ of the body $i$ is denoted as the element $i j$ (Fig. 57). We associate to element $i j$ a density variable $\rho_{i j}$ representing the density of the material present in this element. For the topology optimization problem, variables $\rho_{i j}$ grouped into the vector $\boldsymbol{\rho}$ represent its decision variables. Densities $\rho_{i j}$ equal to 1 are associated with the presence of materials while densities at 0 represent voids.

A challenge in topology optimization is to avoid converging towards a final link design with too many intermediate densities $\left(0<\rho_{i j}<1\right)$ that are difficult to manage by the designer. Thus, in order to solve this issue, an interpolation scheme used in order to define an artificial material is usually introduced into the finite element model. This approach is named the Solid Isotropic Material with Penalization (SIMP, [60]) and it has proven to be efficient. The SIMP scheme used to parameterize the Young's modulus value $E_{i j}$ of the element $i j$ is expressed by:

$$
E_{i j}=E_{\min }+\rho_{i j}^{p}\left(E_{0}-E_{\min }\right), \text { with } \rho_{i j} \in[0,1]
$$

where $p$ is a penalty factor (typically $p=3$ or 4 ), $E_{0}$ is the nominal elasticity modulus of the material and $E_{\min }$ is a very small number. $E_{\min }$ is assigned to regions with voids in order to avoid a loss of regularity of the link or structure stiffness matrix.

Then, based on this definition of the elasticity modulus for the element $i j$, it is possible to build its stiffness matrix (typically based on QUA4 element formulation for planar problems, HEXA8 for 3D). Once all elementary matrices are defined, they can be assembled in order to compute the links and robot stiffness matrices [54].

Finally, based on these behavioral models, the elastic performance of the robot can be characterized: in this work, we only consider the deformations $\mathbf{u}_{e}$ of the five-bar mechanism at its end-effector due to a wrench $\mathbf{f}_{e}$ applied on it. A deformation model can thus be computed and it will take the 
following form:

$$
\mathbf{f}_{e}=\mathbf{K}_{e}\left(\boldsymbol{\pi}_{E}, \mathbf{q}\right) \mathbf{u}_{e}\left(\boldsymbol{\pi}_{E}, \mathbf{q}\right) \Rightarrow \mathbf{u}_{e}\left(\boldsymbol{\pi}_{E}, \mathbf{q}\right)=\mathbf{K}_{e}^{-1}\left(\boldsymbol{\pi}_{E}, \mathbf{q}\right) \mathbf{f}_{e}
$$

in which $\mathbf{K}_{e}\left(\boldsymbol{\pi}_{E}, \mathbf{q}\right)$ is the reduced stiffness matrix characterizing the overall stiffness of the assembled robot at its endeffector [61,56]. This matrix depends of:

- the configuration $\mathbf{q}$ of the robot,

- the Young's moduli $E_{0}$ of the links (which can have different values on the four robot links) which are grouped into the vector $\boldsymbol{\pi}_{E}$. In our case, this vector has a dimension of 4 .

It should be mentioned that the equations leading to the expression (7) are not detailed, but they can be found using the approach in [56].

\subsection{Modeling of the variability in the robot deforma- tions due to the use of wood}

The robot links will be manufactured in acetylated wood, as mentioned in Section 2.2. Wood is an orthotropic material. Accordingly, its elastic behavior is thus characterized by three Young's moduli $\left(E_{L}, E_{R}\right.$ and $\left.E_{T}\right)$, six Poisson's ratios $\left(v_{L R}, v_{L T}, v_{R T}, v_{R L}, v_{T L}, v_{T R}\right)$ and three shear moduli $\left(G_{L R}, G_{L T}, G_{R T}\right)$. However, we decided to model wood like an isotropic materials for the two following reasons:

- the links of parallel robot presents a slenderness corresponding in the longitudinal direction of the wood. Thus they mostly resist to bending moment, like Bernoulli beams would do. As a result, only the longitudinal Young's modulus $E_{L}$ and the mean value of the Poisson's ratio in the two transversal direction $(R$ and $T)$ $v=\frac{\left(v_{L R}+v_{L L}\right)}{2}$ could characterize the link behavior.

- even if the wood is orthotropic, we have the possibility during the manufacture phase to properly design links so that they have a behavior close to the isotropy. This adequate design will be explained in Section 5

Acetylated beech presents a normal distribution of its longitudinal Young's modulus [62]. Let us define as $\mathbb{E}($. the expectation operator and $\sigma($.$) the standard deviation op-$ erator: for the Young's modulus $E$ of the acetylated beech, $\mathbb{E}(E)=\bar{E}=12772 \mathrm{MPa}$ while its standard deviation is equal to $\sigma(E)=2043.5 \mathrm{MPa}$ [63]. The Poisson's ratio $v$ is considered to be constant and equal to 0.3 [26].

Thanks to this characterization of the variability of the beech elastic behavior, it is possible to calculate the expectation vector $\mathbb{E}\left(\mathbf{u}_{e}\right)$ and the covariance matrix $\operatorname{Cov}\left(\mathbf{u}_{e}\right)$ associated with the end-effector deformations $\mathbf{u}_{e}$, by using the approximated equations provided in [30], which have been obtained thanks to a Taylor series expansion at the second order of the expression of $\mathbf{u}_{e}$ with respect to the components $\boldsymbol{\pi}_{E}$ :

$$
\mathbb{E}\left(\mathbf{u}_{e}\right)=\mathbf{u}_{e 0}+\frac{1}{2} \sum_{i} \sum_{j} \mathbf{u}_{e i j} \sigma_{i j}
$$

$$
\begin{aligned}
\operatorname{Cov}\left(\mathbf{u}_{e}\right)= & \sum_{i} \sum_{j} \mathbf{u}_{e i} \mathbf{u}_{e j}^{T} \sigma_{i j} \\
& +\frac{1}{2} \sum_{i} \sum_{j} \sum_{k}\left(\mathbf{u}_{e i} \mathbf{u}_{e j k}^{T}+\mathbf{u}_{e i j} \mathbf{u}_{e k}^{T}\right) \sigma_{i j k} \\
& +\frac{1}{4} \sum_{i} \sum_{j} \sum_{k} \sum_{l} \mathbf{u}_{e i j} \mathbf{u}_{e k l}^{T}\left(\sigma_{i j k l}-\sigma_{i j} \sigma_{k l}\right)
\end{aligned}
$$

where

- $\mathbf{u}_{e 0}$ is the end-effector deformation provided by the equation (7) and estimated when the vector of Young's moduli $\pi_{E}$ is equal to $\pi_{E 0}=[\bar{E} \bar{E} \bar{E} \bar{E}]^{T}$,

- $\mathbf{u}_{e i}=\left.\frac{\partial \mathbf{u}_{e}}{\partial E_{i}}\right|_{\pi_{E 0}}, \mathbf{u}_{e i j}=\left.\frac{\partial^{2} \mathbf{u}_{e}}{\partial E_{i} \partial E_{j}}\right|_{\pi_{E 0}}$, where $E_{i}\left(E_{j}\right.$, resp. $)$ is the elasticity modulus of the link $i$ ( $j$, resp.) of the robot. Detailed expressions of $\mathbf{u}_{e i}$ and $\mathbf{u}_{e i j}$ are provided in the paper [30];

- $\sigma_{i j}=\mathbb{E}\left[\left(E_{i}-\bar{E}\right)\left(E_{j}-\bar{E}\right)\right]$,

- $\sigma_{i j k}=\mathbb{E}\left[\left(E_{i}-\bar{E}\right)\left(E_{j}-\bar{E}\right)\left(E_{k}-\bar{E}\right)\right]$,

- $\sigma_{i j k l}=\mathbb{E}\left[\left(E_{i}-\bar{E}\right)\left(E_{j}-\bar{E}\right)\left(E_{k}-\bar{E}\right)\left(E_{l}-\bar{E}\right)\right]$

It should be mentioned that we tested the validity of the approximated expressions (8) and (9) and the results showed that, for the elasticity parameters of the acetylated beech, the error of approximation is lower than $10 \%$.

Based on these expressions, we defined the following constraints:

$$
u_{d}\left(\mathbf{f}_{e}, \mathbf{q}, k\right)=\mathbb{E}\left(\left\|\mathbf{u}_{d e}\right\|\right)+k \sigma\left(\left\|\mathbf{u}_{d e}\right\|\right) \leq u_{\max }
$$

in which

- $\mathbf{u}_{d e}$ are the components of $\mathbf{u}_{e}$ characterizing the endeffector translational deflections due to the loading $\mathbf{f}_{e}$,

- the function $\sigma\left(\left\|\mathbf{u}_{d e}\right\|\right)$ provides the standard deviations of the end-effector translational deflections.

- $k$ is a positive real number.

We derivated the constraints (10) from the well-known Bienaymé-Tchebichev theorem. This theorem states that, for a stochastic variable $X$, the probability to have $|X-\mathbb{E}(X)|$ $\geq k$ is smaller than $\frac{\sigma(X)^{2}}{k^{2}}$. Consequently, by increasing the value of $k$ in (10), we decrease the probability to have a robot whose deformations can be larger than $u_{\text {max }}$ or $\theta_{\text {max }}$. For instance, with $k=3$, the probability to have a robot whose deflections are smaller than $u_{\text {max }}$ is around $90 \%$.

\subsection{Finding the optimal robot link shapes: Problem for- mulation and results}

Problem formulation: In order to accelerate the optimization process, the shapes of both proximal links are imposed to be identical. Additionally, in order to have a good observation of the distal links, it was decided in the previous sections that the distal links must be cylindrical with a diameter of $80 \mathrm{~mm}$. These links are indeed solicited in tension/compression only when efforts are applied in the plane 


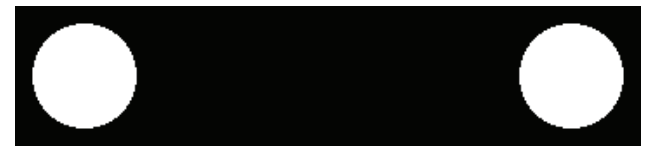

Fig. 6. Design of the proximal links: initial design domain

of the robot motion; thus, they have little deformation. So their shape has little impact on the overall robot deflections.

Based on the previous discussions and the design requirements of Tab. 1. we formulated the following topology optimization problem:

$$
\begin{array}{ll}
\min _{\boldsymbol{\rho}} & z z_{1 R}=z z_{1}+m_{2} \ell_{1}^{2} \\
\text { under } g_{1 i}=\left(u_{d}^{2}\left(\mathbf{f}_{i}, \mathbf{q}^{*}, k\right)-u_{\text {max }}^{2}\right) / u_{\text {max }}^{2} \leq 0, \quad i=1,2
\end{array}
$$

in which

- the objective function $z z_{1 R}=z z_{1}+m_{2} \ell_{1}^{2}$ is a grouping of robot dynamic parameters $\left(z z_{1}\right.$ is the moment of inertia around $z_{0}$ at point $A_{1}$ of the proximal link while $m_{2}$ is the mass of the distal link). This term is usually preponderant in the dynamic model of the five-bar mechanism [64]. Thus, even if there is no constraint provided on the robot dynamics performance in Section 2.3 it is worthwhile to minimize this function as it will lead to a positive impact on the robot dynamic properties (lower input torques, energy consumption, etc).

- $\mathbf{q}^{*}$ are exciting configurations for the calculation of the robot deflections under the loadings $\mathbf{f}_{i}(i=1,2$ - these loadings are defined in Tab. 11). Configurations $\mathbf{q}^{*}$ are the configurations where the robot deflections are the worst, thus ensuring that, if the constraint is respected for the configuration $\mathbf{q}^{*}$, the constraints are respected in the whole dextrous rectangular workspace.

Configurations $\mathbf{q}^{*}$ can be detected using the methodology of [56].

We solved this problem by using the solver LM [65, 56].

Results of optimization: The initial design domain of the proximal links is shown in Fig. 6. The links have two empty holes of $6 \mathrm{~cm}$ in diameter at their extremities for inserting joints (motor shaft at points $A_{i}$, and passive revolute joints at points $B_{i}$ ). Each link is meshed with 22152 QUA4 planar elements of size $1 \times 1 \mathrm{~mm}$ and with thickness of $50 \mathrm{~mm}$. Matlab in the Windows 7 environment has been used in order to encode all models and optimization algorithms. For a fixed value of $k$, calculation time was around $5000 \mathrm{sec}$ (for a Pentium $42.70 \mathrm{GHz}, 16 \mathrm{~GB}$ of RAM).

The value of the objective $z z_{1 R}$ as a function of the parameter $k$ is shown in Fig. 7. The variations in the link design, for increasing values of $k$, are essentially an addition of the material thickness near the insertion hole at the "righthand-side" of the link (hole for inserting the passive joint for the connection with the distal link) and the increase of the presence of material near the "left-hand-side" of the link (hole for inserting the motor shaft). The total inertia value between results for $k=2$ and $k=5$ is almost multiplied by 2 , thus increasing the robustness of the link deflections with respect to the wood elasticity variability, but also degrading the robot dynamic performance.

We decided to finally achieve a prototype based on the results obtained for $k=3$, i.e. for having a probability of $90 \%$ that the deformation will be under the given threshold. The next Section introduces the prototype which has been manufactured based on the previous results, and the characterization of its performance.

\section{Prototype of the wooden industrial robot}

Based on the previous optimization results, a prototype of a wooden five-bar mechanism was designed (Fig. 9p. In this section, some key technological solutions are first highlighted. Then, the implementation of the controllers is discussed. Finally, deformations and accuracy performance are tested.

\subsection{Key mechanical components of the prototype}

In this subsection, we make a focus on the practical achievement of some key technological solutions that have been applied for the final robot design. The major points are for the design of:

- The wooden distal links: they are cylindrical for simplifying their observation by the camera. They are partially hollowed out to decrease their mass.

- The wooden proximal links: they are based on the results of the previous sections on optimal link design. They are achieved from seven laser cut layers $(7 \mathrm{~mm}$ of thickness), assembled together with polyurethane glue. In order to improve their elastic behavior along all directions, the layers are assembled like for a composite material, by differently orienting the wood fibers for each layer. This would make a wooden body with properties as close as possible from the isotropy.

From top to bottom, angles between longitudinal axis of the link and wood grain are $0^{\circ} ; 45^{\circ} ;-45^{\circ} ; 0^{\circ} ;-45^{\circ}$; $45^{\circ} ; 0^{\circ}$. CAD design of the proximal links is shown in Fig. 8(a) Differences between the CAD model and the topology optimization results are due to local edge distances needed around the holes to ensure wood resistance, but the truss-like shape is kept.

- The wooden chassis: this part is made with acetylated pine, and it supports two direct drive actuators (SIMOTTICS S-1FL6 servomotors) as depicted in Fig. 8(b).

- The passive revolute joints: high precision angular contact ball bearings have been bought to design the revolute joints. This configuration delivers a high level of stability and minimal backlash for the assembly. Additionally, the revolute joint axes have a diameter of $20 \mathrm{~mm}$. They have been deliberately oversized in order to avoid that they have an impact the measurements on the deformations of the wooden links.

Even if we do not present them in this paper because 


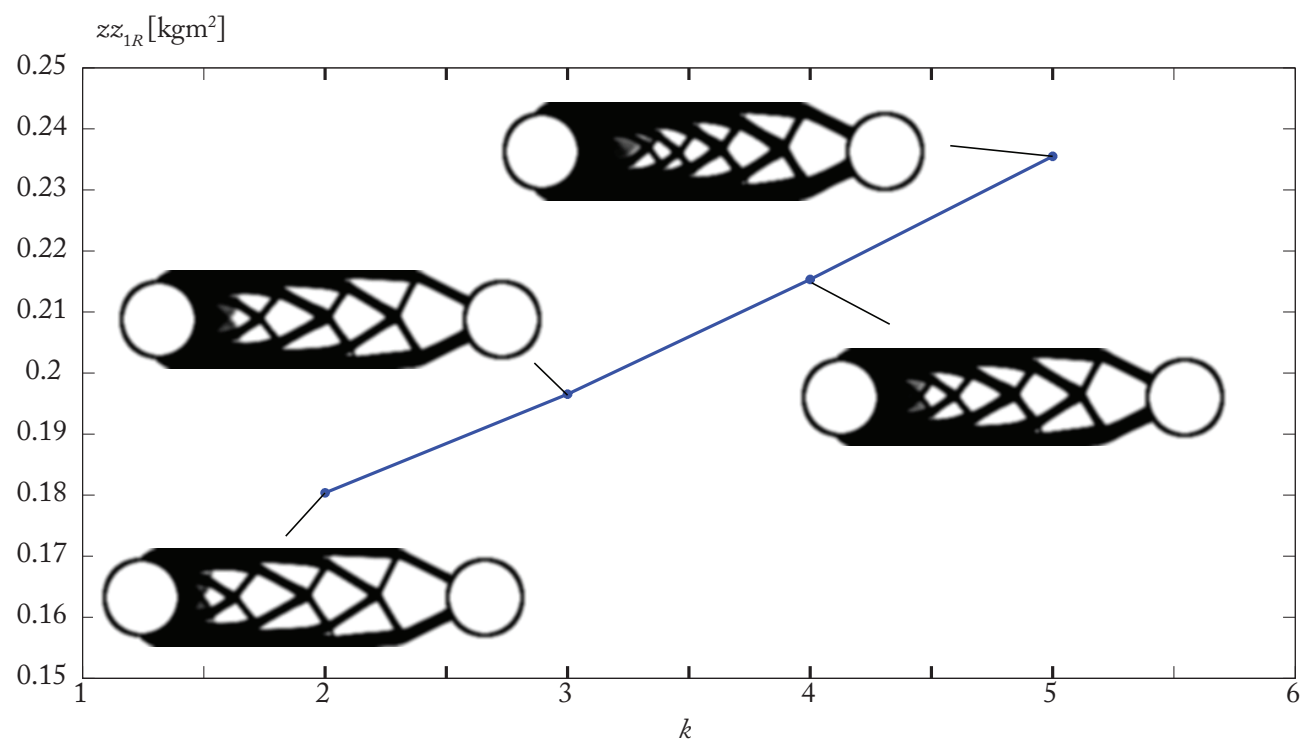

Fig. 7. Design of the proximal links: final results for different values of $k$

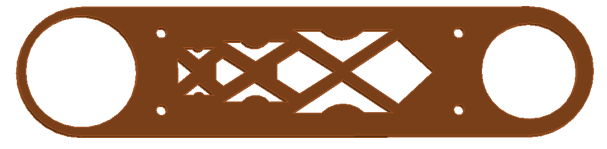

(a) Proximal Link: CAD Design

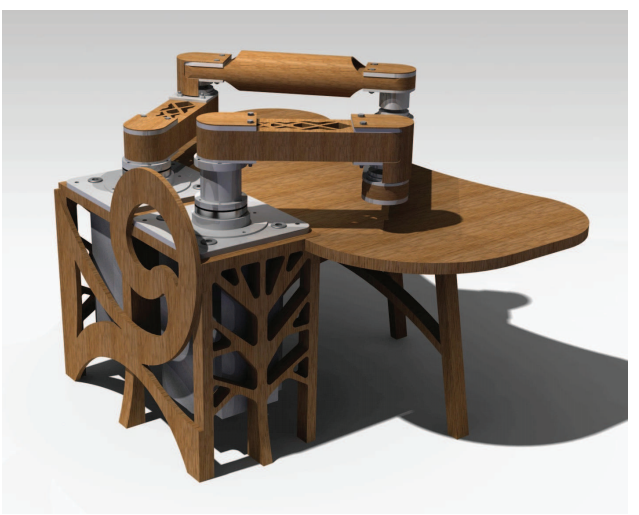

(b) Final Prototype

Fig. 8. CAD Design of a wooden five-bar mechanism.

they are only little informative, FEA simulations with the CAD model have been carried out in order to check the overall deformation of the robot. End-effector deflections under a loading of $20 \mathrm{~N}$ along $z_{0}$ are estimated at 400 microns for the nominal value of the beech Young's modulus, which seems acceptable to us.

Based on this CAD model, a prototype of the robot, named RobEcolo, was entirely manufactured by the technical staff of LS2N and École Supérieure du Bois (Fig. 9). The next Section is on the implementation of the robot controller.

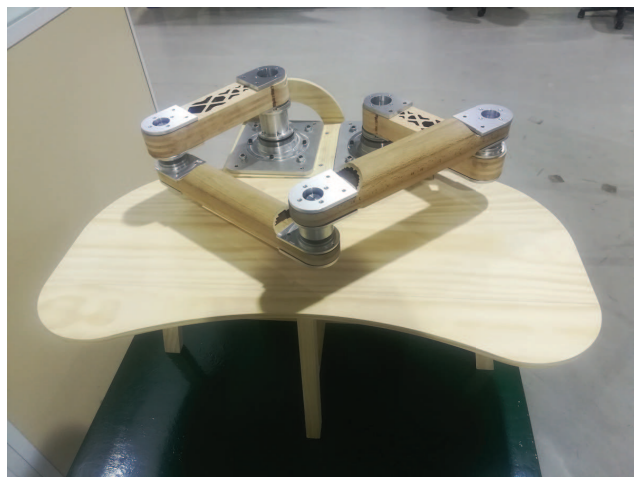

Fig. 9. Final prototype of the RobEcolo robot.

\subsection{Controller implementation}

Communication between the camera, motor, and controller: The connections between the camera, the motors and the control board are depicted in Fig. 10. The robot's motors inputs are provided by the control board (and through the converters). This control board (a dSpace1103) is interfaced with the robot's computer in order to communicate the desired velocities to the motors (obtained by the high-level vision-based controller defined in Section 3.1 which are converted by a low-level computed-torque controller [42] into input torques control references, as it was done in [66 67]. Additionally, the control board also communicates and processes the information extracted and sent from the cameras by the cameras' computer. While the communication between the robot's computer and the control board is done through fiber optics, between the later and the cameras' computer only a serial port RS232 is available, which highly limits the amount of information that can be transmitted. Moreover, the data acquisition frequency of camera is limited at $165 \mathrm{~Hz}$. Globally, with the necessary image processing for extracting primitives for 


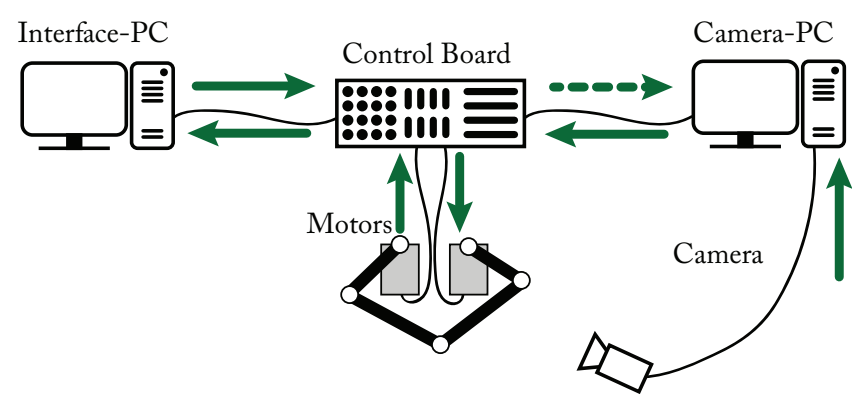

Fig. 10. Interaction layout for Robecolo prototype.

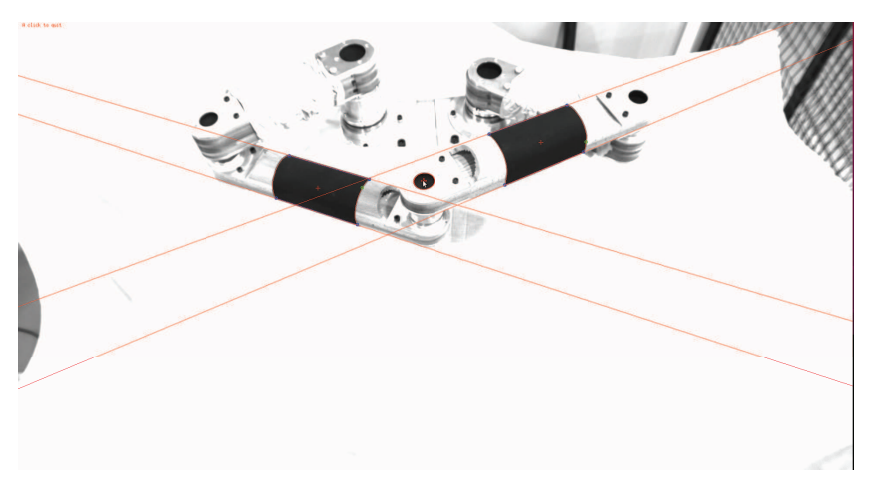

Fig. 11. Tracker for detecting leg edges and end-effector location.

the controller, the sampling rate for the robot vision-based (high-level) controller is at $50 \mathrm{~Hz}$, while the (low-level) computed-torque controller has a sampling rate of $1 \mathrm{kHz}$.

Camera calibration and image processing: For the controller based on the observation of the end-effector, the position and orientation of the camera has been selected at $\left\{x_{c}=0 \mathrm{~m}, y_{c}=0.35 \mathrm{~m}, z_{c}=0.8 \mathrm{~m}, \phi=0 \mathrm{rad}, \theta=\pi \mathrm{rad}, \psi=\right.$ $0 \mathrm{rad}$ \} so that the workspace can be observed in totality while the distance of the camera to the plane of motion is as small as possible. For the controller based on the observation of the links, the camera is located at the optimized configuration provided in Tab. 3 .

Intrinsic parameters of the camera have been calibrated by assuming that its geometry properties can be modeled using the standard pin-hole camera model [35]. The calibration process used in the available VISP library which implements a camera calibration based on virtual visual servoing, presented by [68,69].

The VISP libraries offer an edge tracker with a subpixelic algorithm. However, due to the environment conditioning (large lightning variations), it could not be used. A dedicated tracker was implemented, which tracks the end-effector and the leg edges (Fig. 11). Distal links have been covered with black paper sheets in order to facilitate their detection by the camera.

The camera measurement noise has been estimated. In order to measure the real noise introduced by the camera, the camera and robot data were recorded while placing the later on different points of the workspace of interest (see Fig. 12). The methodology to measure the noise was:

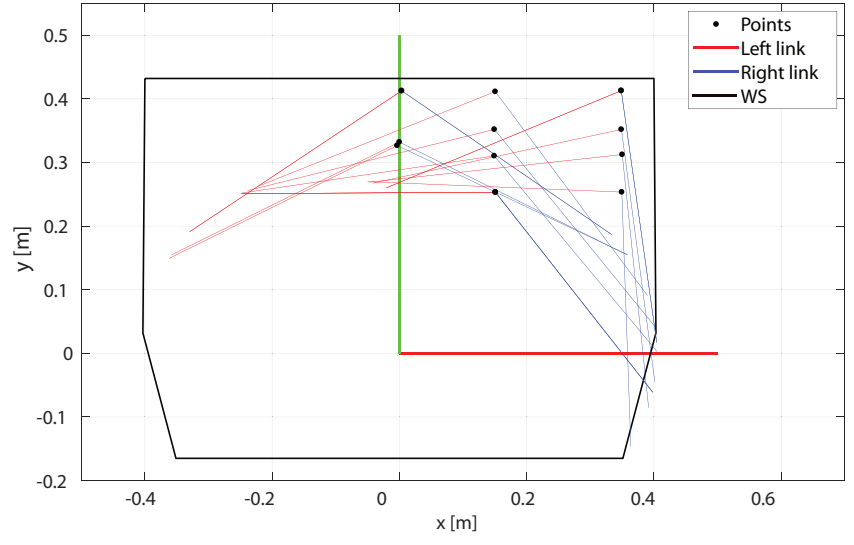

Fig. 12. Robot links configuration in the noise analysis points.

1. Move the robot to the desired point,

2. Deactivate the controller so that the robot is sure to stay fixed,

3. Measure the data for $20 \mathrm{~s}$ at $50 \mathrm{~Hz}$,

4. Repeat items 1 to 3 for all the points.

Maximal values for the noise measured when detecting a point located at the end-effector is around 0.025 pixel, i.e. 4 times lower than the noise used in the error model. For the observation of the link projections, the maximal noise is much larger, around 0.4 pixel, i.e. 4 times bigger than the noise used in simulation. It was indeed difficult to quantify at the beginning of the design process what could be the noise coming from the extraction of the leg. This will have necessarily an impact on the final robot accuracy with the line-based controller (case B).

Controller modifications: The two types of controllers defined in Section 3.1 have been transposed into the joint space. In order to hasten the convergence towards the desired measurements $\mathbf{s}^{*}$, strategies for adaption of the controller gain $\lambda$ have been used [70]. In order to avoid having discontinuity of velocities at the beginning of the motion, input-smoothing technique proposed in [71] was used.

Next Section deals with the characterization of the performance of the prototypes in terms of deformations and positioning accuracy.

\subsection{Prototype performance \\ 5.3.1 Deformations}

Experimental setup: In order to test the robot performance in deformation, the prototype was submitted to different efforts applied on its end-effector and the deformations were measured.

The deformations were measured in several points of the workspace. Particularly, five points located at the extremities of the rectangular workspace and at the center of this (points A, B, C, D and E in Fig. 47). Loads were then applied along the three main axes of the robot frame $x_{0}, y_{0}$ and $z_{0}$.

The material used to perform this experiment is composed of: 


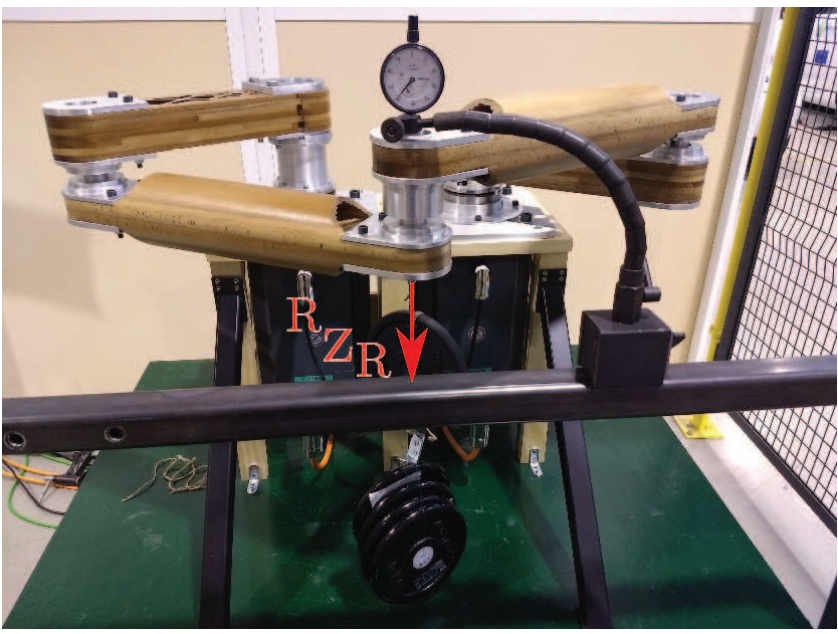

(a) Tools disposition to measure deformations along the vertical axis

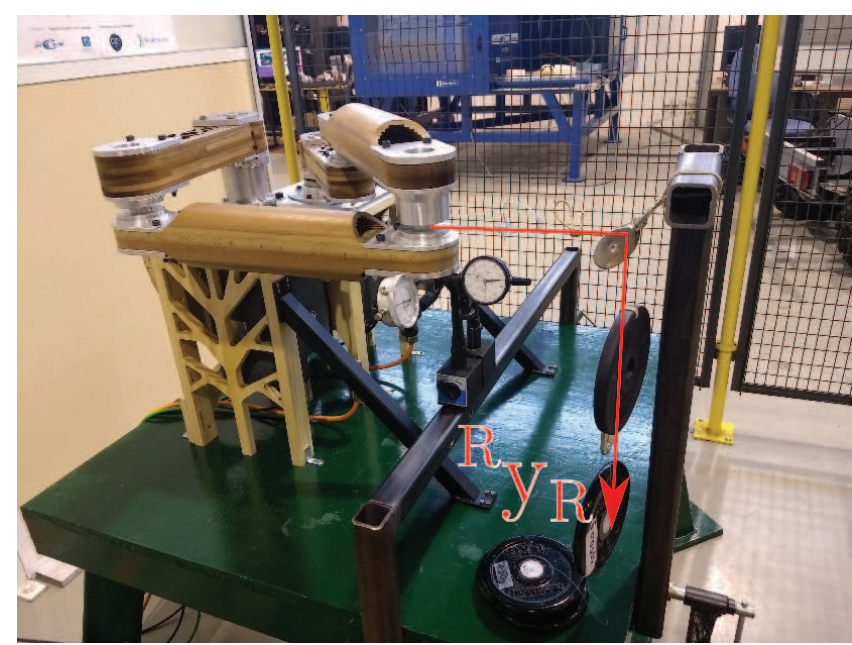

(b) Tools disposition to measure deformations in the horizontal plane

Fig. 13. Benchmark for measuring the robot deformations

- measurement devices, Mitutoyo dial micrometers (ana$\log$ comparator) with a resolution of $0.01 \mathrm{~mm}$, mounted on magnetic bases to hold them on place. A rectified square is attached to the end-effector and served as a contact surface for taking the measurements with the micrometers.

- a device composed of a mass payload that can be attached to a wire connected to a pulley so that it becomes possible to exert a constant force in the horizontal plane at the robot end-effector (Fig. 13).

The process followed for each measurement of the deformation, along a single axis of the robot frame (either $\boldsymbol{x}_{0}$, $\boldsymbol{y}_{0}$ or $\boldsymbol{z}_{0}$ ) was as follows:

1. Apply a pre-charge of $1 \mathrm{~kg}$ in order to compensate any possible existing wobbliness induced by the joints. The next step can start when the measured deformation is stabilized (less than $0.01 \mathrm{~mm}$ deformation over a minute, two minutes are usually necessary to reach this state),

2. Apply a payload of $n \mathrm{~kg}(n=1,2,3,4)$,

3. Measure with a micrometer the deformations for each
Table 4. Mean of the norm of the deformations in the plane $\left(x_{0} O y_{0}\right)$ in $\mathrm{mm}$ for a horizontal load of $20 \mathrm{~N}$.

\begin{tabular}{l|lllll}
\hline \hline & $\begin{array}{l}\text { Point } \\
\text { A }\end{array}$ & $\begin{array}{l}\text { Point } \\
\text { B }\end{array}$ & $\begin{array}{l}\text { Point } \\
\text { C }\end{array}$ & $\begin{array}{l}\text { Point } \\
\text { D }\end{array}$ & $\begin{array}{l}\text { Point } \\
\text { E }\end{array}$ \\
\hline Load along $\boldsymbol{x}_{0}$ & 0.04 & 0.16 & 0.09 & 0.16 & 0.07 \\
\hline Load along $\boldsymbol{y}_{0}$ & 0.03 & 0.08 & 0.09 & 0.09 & 0.12 \\
\hline \hline
\end{tabular}

Table 5. Mean of the vertical deformations in $\mathrm{mm}$ for a vertical load of $20 \mathrm{~N}$.

\begin{tabular}{lllll}
\hline \hline Point A & Point B & Point C & Point D & Point E \\
\hline 0.33 & 0.40 & 0.32 & 0.40 & 0.21 \\
\hline \hline
\end{tabular}

payload. It is important to note that, the measures must be taken right after the payload application, before the viscoelastic behavior of the wood influences the measurements,

4. Remove the $n$-kg mass; wait two minutes before applying a new load, so that the robot came back to its initial deformation state (verification on the micrometers),

5. Repeat steps 2 to 4 five times for each configuration, for each axis of loading.

Because of the large number of necessary measurements (4 payloads applied in 3 directions for 5 points in the workspace), only two measurements have been be taken per experiment. We take the mean value of them for characterizing the robot deformation.

Results: Tables 4 and 5 show a summary of the experimental results. It is possible to see that the maximal deformation in the horizontal plane under $20 \mathrm{~N}$ loading is lower than 200 microns (which was the expected deformation) while the deformation under the same loading but along the vertical axis is limited to 400 microns.

\subsubsection{Positioning accuracy}

Experimental setup: In order to estimate the robot accuracy, the precision and repeatability of the robot were measured.

It is important to note that, the precision measurements correspond to the relative precision of the robot. Indeed, since an IVBS approach was implemented [35], the control error is measured in the image frame and there is no reconstruction of the robot 3D geometry. Hence, the position is known relative to the image frame and not to the robot frame. However, this is not an issue if all tasks are referred in the image frame, as usually done in visual servoing.

The precision and repeatability experiment were based on the norm ISO9283, so five points in the workspace were chosen and a trajectory approaching them from all the possible main directions was performed (along $\boldsymbol{x}_{0}, \boldsymbol{y}_{0}$ and with a combination of both motions - Fig. 14. From the previous layout, 17 trajectories were performed and the measurements followed the following methodology: 


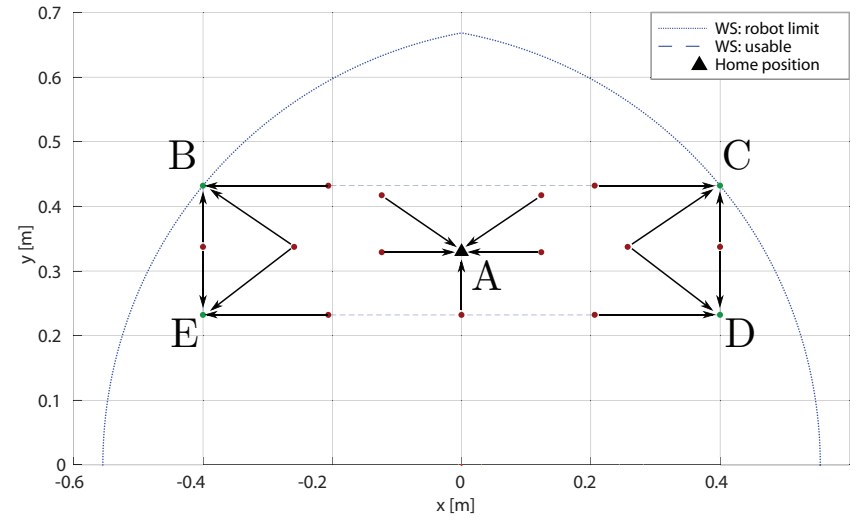

Fig. 14. Trajectories for testing the robot accuracy: the accuracy is tested in points $A, B, C, D$ and $E$, red points represent the initial locations for the test trajectories.

Table 6. Repeatability measurements in microns for the two types of controllers.

\begin{tabular}{l|lllll}
\hline \hline Controller & $\begin{array}{l}\text { Point } \\
\text { A }\end{array}$ & $\begin{array}{l}\text { Point } \\
\text { B }\end{array}$ & $\begin{array}{l}\text { Point } \\
\text { C }\end{array}$ & $\begin{array}{l}\text { Point } \\
\text { D }\end{array}$ & $\begin{array}{l}\text { Point } \\
\text { E }\end{array}$ \\
\hline Case A & 58 & 49 & 54 & 40 & 61 \\
\hline Case B & 480 & 598 & 167 & 270 & 456 \\
\hline \hline
\end{tabular}

1. Place the robot in the desired final position using the position controller,

2. Set the comparators and the visual features. Read the initial comparator measures;

3. Move the robot to the initial position of the trajectory,

4. Return to the desired position using the visual servoing controller,

5. Read the measures in the comparator:

(a) Repeat 20 times the steps from 3 to 5 using the end effector as a feature,

(b) Repeat 20 times the steps from 3 to 5 using the edges as a feature.

6. Repeat all the steps above for the 17 trajectories,

7. Repeat all the steps above for the two tested controllers.

Results: Tables 6 and 7 show a summary of the experimental results. It appears that the controller based on the observation of the end-effector is the best (positioning accuracy lower than 40 microns, repeatability lower than 60 microns), for two main reasons:

1. the measurement noise is very low, as shown above,

2. it is less sensitive to any type of unmodeled effects.

Because the measurement noise is much higher for the line-based controller, this lead to worse accuracy performance. The repeatability is under 600 microns for the tested configurations, which is not that far from the desired 500microns repeatability requested in Tab. 1. but the positioning accuracy is twice as large. However, we succeed at least with one of the two controllers to have accuracy lower than
Table 7. Positionning accuracy measurements in microns for the two types of controllers.

\begin{tabular}{l|lllll}
\hline \hline Controller & $\begin{array}{l}\text { Point } \\
\text { A }\end{array}$ & $\begin{array}{l}\text { Point } \\
\text { B }\end{array}$ & $\begin{array}{l}\text { Point } \\
\text { C }\end{array}$ & $\begin{array}{l}\text { Point } \\
\text { D }\end{array}$ & $\begin{array}{l}\text { Point } \\
\text { E }\end{array}$ \\
\hline Case A & 38 & 27 & 21 & 22 & 28 \\
\hline Case B & 273 & 858 & 76 & 978 & 398 \\
\hline \hline
\end{tabular}

500 microns.

In the next Section, we perform some discussions on the experimental results and on the final environmental impact of our robot.

\section{Discussions}

\subsection{Discussion about the impact of the wooden robot di-} mensional variability

As mentioned in the introduction, wood will endure dimensional variations due to hygrothermal evolution of the environnement. In order to cope with this issue, we proposed to implement sensor-based controllers so that the positioning accuracy can be ensured.

Thus, it would have been of interest to perform tests in climatic chambers in order to validate this hypothesis. However, these tests have not been made for the following reason. Indeed, the controller based on the observation of the end-effector is totally independent of any robot geometric parameters. So its accuracy performance is theoretically totally not affected by the change of the robot lengths. In practice, results with little differences may occur because of slight overconstraints appearing in the robot mechanical architecture due to changes of link dimensions, and thus leading to potential backlash issues. However, changes of link dimensions are unlikely to lead to strong differences in the accuracy performance of this controller, especially when initial results showed an accuracy lower than 60 microns (8 times lower than the desired maximal accuracy).

Therefore, even if these tests have not been performed, we believe that accuracy of a wooden robot can be guaranteed thanks to the use of an appropriate sensor-based controller.

\subsection{Discussion about the impact of the wood mechanical property variability for the robot mechanical design}

Even if we succeeded in creating a prototype with deformations lower than 500 microns under $20 \mathrm{~N}$ loadings, as expected from the requirements, because the coefficient of variation for the mechanical properties is of $15 \%$ for the beech we used, it is difficult to give a definitive conclusion on the validity of our reliable design approach: for this, it would be necessary to create a large number of prototypes, and to check their deformations for all. This is obviously impossible to achieve within a reasonable time and money expense.

In order to partially overcome this problem, we performed the following additional simulations based on a 
Table 8. Number of robots with unacceptable deformations among the 50000 tested designs

\begin{tabular}{lllll}
\hline \hline Selected robot designs & $k=2$ & $k=3$ & $k=4$ & $k=5$ \\
Number of robots with & 160 & 53 & 29 & 10 \\
$\begin{array}{l}\text { unacceptable deformations } \\
\text { Percentage of robots with }\end{array}$ & 0.32 & 0.11 & 0.06 & 0.02 \\
\begin{tabular}{l} 
unacceptable deformations \\
\hline
\end{tabular}
\end{tabular}

Monte Carlo approach. For all final robot designs provided in Fig. 77, from $k=2$ to $k=5$, we randomly set 50000 different Young's moduli per link (so a total of 200000 different Young's moduli) that could occur based on the variability law of the beech: the Youngs moduli are computed based on a Gaussian law with an expectation of $12772 \mathrm{MPa}$ and a standard deviation equal to $2043.5 \mathrm{MPa}$.

Then, for a given value of $k$, we computed the maximal deflections in the dextrous workspace of the 50000 possible robot designs under the loadings defined in Tab. 1. Results are shown in the Tab. 8

Simulation results, based on the use of a wood deformation model defined in [63], which is able to predict link deformation with an average error of $10 \%$, showed that, even in the less constrained case in terms of reliability of the design $(k=2)$, the percentage of robots with unacceptable deflections is lower than $0.5 \%$. This showed that the proposed reliable topology optimization algorithm, based on the definition of probabilistic constraints, leads to the definition of link designs that are able to properly constrain in simulation the risk of having designs with unacceptable rigidity. Therefore, we can expect that the risk to have real designs with unacceptable deformations is very low.

\subsection{Discussion about the impact of the use of the wood on the robot dynamics properties}

As already explained in Section 2, the RobEcolo robot is a prototype that has been designed for validating performance in terms of stiffness and accuracy, which are necessary steps, but challenging enough, in order to prove the validity of using wood for robot design purpose. Therefore no dynamic properties have been imposed, even if the design process tended to reduce the robot link inertia.

However, we are aware that using wood instead of metal may have an impact on the robot dynamics. We may expect an increase in the mass and inertia may lead to an increase of the robot input torques, and thus to an increase of the power consumption. However, in case this will appear, input torques reduction techniques or energy saving techniques could be applied, like balancing techniques [4.72], optimal trajectory planning [73], etc. Large vibrations could occur when using wooden links. However, the ratio stiffness-todensity of the wood is close to the one of iron or aluminum alloys [26], which makes us think that the problem of increased vibrations will be limited. However, in case vibration issues appear, several strategies could be used, like de- signing a robot under the objective of maximizing the natural frequencies [38], using active or passive vibration control techniques [74, 75]. Investigating the impact of designing a robot in wood on its dynamics properties is left as part of our future works.

\subsection{Discussion about the wooden robot Life Cycle As- sessment}

We claimed in the introduction that we wanted to use wood for robot mechanical design in order to lower the environmental impact of the mechanical robot architecture design. In order to validate this hypothesis, a Life Cycle Assessment (LCA) was performed on the mechanical architecture of our robot [76]. This LCA of the RobEcolo prototype is a technical result that will not be detailed in this paper, but we summarize what is inside this report, that is accessible online.

In this report, only the impact of the manufacture of the mechanical architecture of the wooden robot is modeled, and compared with a five-bar mechanism made of steel. The following assumptions were made:

- The steel-made robot has the links lengths and the same mass. The assumption on the mass was made because, as mentioned in introduction, the rigidity-to-density ratios of typical wood and steel are almost identical. So, for the same rigidity properties, the steel-made robot should have almost the same mass as the wooden robot.

- Only the wooden robot needs a camera for precise positioning. We considered that the repeatability of the steelmade robot can be obtained by model-based controllers.

- Both robots required machining processes for manufacturing their links. We decided to model the same machining tasks for both robots. This choice may be not fair, but even if the steel-made robot could need less tasks, the wood is a soft material (compared with steel) which needs less energy for being manufactured.

- The impacts of the electronic part, except the camera, were not modeled because they would be equivalent for both types of robots.

The report shows that, with respect to a steel-made robot, our robot is better in global warming impact, use of fossile fuels (wood comes our forests and we consume less petrol than with metals coming from the extremity of the world) and terrestrial ecotoxicity (15 to $30 \%$ of reduction). However, other impact criteria related to environment acidification and water toxicity are worst: this is because of the acetylation not free of impact for the environment. In order to decrease much more the environmental impact, two directions are proposed in the report: using raw wood instead of acetylated wood, and removing the metal joints which have a non negligible impact, and replacing them by joints made of bio-sourced materials. These two directions are part of our future works, as well as further investigations on the influence of the size of the robot on the gains in terms of environmental impacts. However, even if the results in terms of environmental impact decrease are not satisfactory enough, we 
would like to conclude that with our work, which succeeded to show that designing a wooden robot with performance in terms of siffness and accuracy compatible with Industry requirements is feasible, we provided a first step towards robot eco-sustainable mechanical design.

\section{Conclusion}

In order to try to decrease the environmental impact of robots during their manufacturing phase, this paper investigated the possibility of replacing metal links used in robot design by wooden elements. Wood has indeed an interesting mass-to-stiffness ratio, competitive with metals currently met in robot design, but has also some drawbacks, like significant dimensional and mechanical variabilities. This is an issue when dealing with the design of industrial robots which must usually be accurate and stiff.

In order to be able to guarantee the stiffness and accuracy performance of the wooden robot, an integrated design process has been proposed. It combined (i) proper wood type selection, (ii) the definition of adequate control strategies where external sensors (here, a camera) are used to directly observe the object to be controlled (in our case, the end-effector) and (iii) a robust design approach dealing with wood uncertainties and taking into account at the earliest step the performance of the controller in terms of accuracy: this integrated methodology is called "control-based design" approach.

Based on the use of this integrated design process, a prototype of a wooden five-bar mechanism was designed and manufactured. The requirements were to design a robot whose repeatability was lower than 500 microns and whose deformation under $20 \mathrm{~N}$ loading was lower than 200 microns in the horizontal plane (500 microns along the vertical axis). Experiments for characterizing its accuracy and its stiffness were performed. We showed that, with a proper visual servoing algorithm in which we directly observed the location of the end-effector, a repeatability of 60 microns was obtained, i.e. 8 times better. For the deformation performance, deformations under a $20 \mathrm{~N}$ load applied on the end-effector were lower than 160 microns in the horizontal plane and $400 \mathrm{mi}-$ crons along the vertical axis. These results are guaranteed in a rectangular workspace of $800 \mathrm{~mm} \times 200 \mathrm{~mm}$.

Finally, we discussed about the remaining environmental impact of our prototype. This environmental impact was decreased for some indices like global warming, but increased for some others like water toxicity because of the use of an acetylated wood for manufacturing the robot. However, with our work, which is a proof of concept that designing a wooden robot with performance in terms of stiffness and accuracy compatible with Industry requirements is realistic, we provided a first step towards robot eco-sustainable mechanical design.

\section{Acknowledgements}

This work was supported by the French Région Pays de la Loire (Project RobEcolo, Convention No. 2015-10773).
The authors would like to thank:

- Mrs. V. Safyannikova for her work on the CAD design of the RobEcolo robot chassis,

- Dr. O. Kermorgant for his support on visual servoing and image processing,

- Dr. R. Chenouard and Mrs. O. Hryshaienko for their help for performing the LCA of the RobEcolo prototype,

- All the technical and engineering staff of our institutions for their technical support.

\section{References}

[1] Carro Fernandez, G., Martin Gutierrez, S., Sancristobal Ruiz, E., Mur Perez, F., and Castro Gil, M., 2012. "Robotics, the new industrial revolution". IEEE Technology and Society Magazine, 31(2), pp. 51-58.

[2] Yang, G., Bellingham, J., Dupont, P., Fischer, P., Floridi, L., Full, R., Jacobstein, N., Kumar, V., McNutt, M., Merrifield, R., Nelson, B., Scassellati, B., Taddeo, M., Taylor, R., Veloso, M., Lin Wang, Z., and Wood, R., 2018. "The grand challenges of Science Robotics". Science Robotics, 3(14), pp. 1-14.

[3] Galitsky, C., and E.Worrell, 2008. Energy efficiency improvement and cost saving opportunities for thevehicle assembly industry. Tech. Rep. LBNL-50939R, Berkeley: U.S. Environmental Protection Agency.

[4] Chaudhary, H., and Saha, S., 2009. Dynamics and balancing of multibody systems. Springer.

[5] Kim, Y., 2017. "Anthropomorphic low-inertia highstiffness manipulator for high-speed safe interaction". IEEE Transactions on Robotics, 33(6), pp. 1358-1374.

[6] Chung, W., Fu, L., and Hsu, S., 2016. Handbook of Robotics, 2nd edition ed. Springer-Verlag, Berlin Heidelberg, ch. 8: Motion Control, pp. 163-194.

[7] T.Chettibi, H.E.Lehtihet, M.Haddad, and S.Hanchi, 2004. "Minimum cost trajectory planning for industrial robots". European Journal of Mechanics - A/Solids, 23(4), pp. 703-715.

[8] Ieropoulos, I., Greenman, J., Melhuish, C., and Horsfield, I., 2010. "EcoBot-III: A robot with guts". In Artificial Life X: Proceedings of the Twelfth International Conference on the Synthesis and Simulation of Living Systems, pp. 733-740.

[9] Mei, Y., Lu, Y., Hu, Y., and Lee, C., 2005. "A case study of mobile robots energy consumption and conservation techniques". In Proceedings of the 12th International Conference on Advanced Robotics, pp. 492-497.

[10] Verstraten, T., Beckerle, P., Furnémont, R., Mathijssen, G., Vanderborght, B., and Lefeber, D., 2016. "Series and parallel elastic actuation: Impact of natural dynamics on power and energy consumption". Mechanism and Machine Theory, 102, pp. 232-246.

[11] Norm ISO 14040, 2006. Environmental management life cycle assessment - principles and framework.

[12] Norm ISO 14044, 2006. Environmental management life cycle assessment - requirements and guidelines. 
[13] Fizians, 2015. Eco-design of two types of robots: KUKA 270 and IRSbot-2. Tech. rep.

[14] Thakur, V., 2013. Green Composites from Natural Resources. CRC Press.

[15] Falk, R. H., 2010. Wood Handbook. United States Department of Agriculture, Madison, Wisconsin, ch. 1: Wood as a sustainable building material, pp. 1-6.

[16] Norm EN 338, 2016. Structural timber strength classes.

[17] Shintake, J., Sonar, H., Piskarev, E., Paik, J., and Floreano, D., 2017. "Soft pneumatic gelatin actuator for edible robotics". In Proceedings of the IEEE/RSJ International Conference on Intelligent Robots and Systems.

[18] Ricotti, L., Trimmer, B., Feinberg, A. W., Raman, R., Parker, K. K., Bashir, R., Sitti, M., Martel, S., Dario, P., and Menciassi, A., 2017. "Biohybrid actuators for robotics: A review of devices actuated by living cells". Science Robotics, 2(12).

[19] Irimia-Vladu, M., Troshin, P. A., Reisinger, M., Shmygleva, L., Kanbur, Y., Schwabegger, G., Bodea, M., Schwdiauer, R., Mumyatov, A., Fergus, J. W., Razumov, V. F., Sitter, H., Sariciftci, N. S., and Bauer, S., 2010. "Biocompatible and biodegradable materials for organic field-effect transistors". Advanced Functional Materials, 20(23), pp. 4069-4076.

[20] Hamann, H., Wahby, M., Schmickl, T., Zahadat, P., Hofstadler, D., Stoy, K., Risi, S., Faina, A., Veenstra, F., Kernbach, S., Kuksin, I., Kernbach, O., Ayres, P., and Wojtaszek, P., 2015. "flora robotica - mixed societies of symbiotic robot-plant bio-hybrids". In Proceedings of the IEEE Symposium on Artificial Life, pp. 11021109.

[21] Laurent, T., Kergueme, J., Arnould, O., and Dureisseix, D., 2010. "Vers un robot en bois: Première partie". Technologies, 168, pp. 28-36. in French.

[22] Laurent, T., Kergueme, J.-L., Arnould, O., and Dureisseix, D., 2010. "Vers un robot en bois. Seconde partie". Technologies, 169, pp. 24-35. In French.

[23] Csiszar, A., Sommer, P., and Lechler, A., 2015. "EcoBotics: Advantages and challenges of building a bamboo robot arm". In Proceedings of the IEEE International Conference on Industrial Technology.

[24] Nguyen, A., Béakou, A., Charlet, K., and Bouzgarrou, B., 2014. "Optimal-design of lightweight mechanism links made of hybrid laminate composite". In Proceedings of the International Symposium on Green Manufacturing and Applications.

[25] NGuyen, A., 2015. "Matériaux composites à renfort végétal pour l'amélioration des performances de systèmes robotiques". $\mathrm{PhD}$ thesis, Institut Pascal, Clermont-Ferrand. in French.

[26] Kretschmann, D., 2010. Ch. 5: Mechanical properties of Wood. Forest Products Laboratory, United States Department of Agriculture Forest Service, Madison, Wisconsin.

[27] Wang, C., and Piao, C., 2011. "From hydrophilicity to hydrophobicity: a critical review". Wood and Fibre
Science, 42(4), pp. 490-510.

[28] Célino, A., Fréour, S., Jacquemin, F., and Casari, P., 2014. "The hygroscopic behavior of plant fibers: a review". Frontiers in Polymer Chemistry, 43(1), pp. 112.

[29] Corbel, D., Company, O., Krut, S., and Pierrot, F., 2010. "Enhancing PKM Accuracy by Separating Actuation and Measurement: A 3DOF Case Study". Journal of Mechanisms and Robotics, 2(3), Aug., pp. 03100081-0310008-11.

[30] Asadpoure, A., Tootkaboni, M., and Guest, J., 2011. "Robust topology optimization of structures with uncertainties in stiffness - Application to truss structures". Computers and Structures, 89, pp. 1131-1141.

[31] Kaci, L., Briot, S., Boudaud, C., and Martinet, P., 2018. "RobEcolo: Optimal design of a wooden fivebar mechanism". In Proceedings of the ASME International Design Engineering Technical Conferences $\&$ Computers and Information in Engineering Conference.

[32] Briot, S., Pashkevich, A., and Chablat, D., 2010. "Technology-oriented optimization of the secondary design parameters of robots for high-speed machining applications". In Proceedings of the ASME International Design Engineering Technical Conferences and Computers and Information in Engineering Conference.

[33] Verco, 2013. Accoya wood 2012 cradle-to-gate carbon footprint update. Tech. rep.

[34] van der Lugt, P., Bongers, F., and Vogtlander, J., 2016. "Environmental impact of constructions made of acetylated wood". In Proceedings of the World Conference on Timber Engineering.

[35] Chaumette, F., and Hutchinson, S., 2008. Ch. 24: Visual Servoing and Visual Tracking. Springer.

[36] Andreff, N., Dallej, T., and Martinet, P., 2007. "Imagebased visual servoing of Gough-Stewart parallel manipulators using legs observation". The International Journal of Robotics Research, 26(7), pp. 677-687.

[37] Briot, S., Rosenzveig, V., Martinet, P., Özgür, E., and Bouton, N., 2016. "Minimal representation for the control of parallel robots via leg observation considering a hidden robot model". Mechanism and Machine Theory, 106, pp. 115-147.

[38] Briot, S., Caro, S., and Germain, C., 2017. "Design procedure for a fast and accurate parallel manipulator". ASME Journal of Mechanisms and Robotics, 9(6), pp. 061012-1-061012-11.

[39] Merlet, J., 2006. Parallel Robots, 2nd ed. Springer.

[40] International federation of robotics (ifr).

[41] Campos, L., Bourbonais, F., Bonev, I., and Bigras, P., 2010. "Development of a five-bar parallel robot with large workspace". In Proceedings of the ASME International Design Engineering Technical Conferences.

[42] Khalil, W., and Dombre, E., 2002. Modeling, Identification and Control of Robots. Hermes Penton London.

[43] Briot, S., and Gautier, M., 2015. "Global identification of joint drive gains and dynamic parameters of parallel 
robots". Multibody System Dynamics, 33(1).

[44] Chaumette, F., and Hutchinson, S., 2006. "Visual servo control part I: Basic approaches". IEEE Robotics and Automation Magazine, 13(4), pp. 82-90.

[45] Andreff, N., Espiau, B., and Horaud, R., 2002. "Visual servoing from lines". International Journal of Robotics Research, 21(8), pp. 679-700.

[46] Chaumette, F., 2004. "Image moments: a general and useful set of features for visual servoing". IEEE Transactions on Robotics, 20(4), pp. 713-723.

[47] Vignolo, A., Briot, S., Martinet, P., and Chen, C., 2014. "Comparative analysis of two types of leg-observationbased visual servoing approaches for the control of the five-bar mechanism". In Proceedings of the Australasian Conference on Robotics and Automation.

[48] Chaumette, F., and Hutchinson, S., 2007. "Visual servo control, part II: Advanced approaches". IEEE Robotics and Automation Magazine, 14(1), pp. 109-118.

[49] Traslosheros, A., Sebastian, J., Angel, L., Roberti, F., and Carelliz, R., 2007. "Visual servoing of a parallel robot system". In Proceedings of the European Control Conference.

[50] Shi, B., Zhao, H., Ben-Ezra, M., Yeung, S., FernandezCull, C., Shepard, R., Barsi, C., and Raskar, R., 2014. "Sub-pixel layout for super-resolution with images in the octic group". In Proceedings of the European Conference on Computer Vision.

[51] Gosselin, C., and Angeles, J., 1990. "Singularity analysis of closed-loop kinematic chains". IEEE Transactions on Robotics and Automation, 6(3), pp. 281-290.

[52] Gosselin, C., and Angeles, J., 1991. "A global performance index for the kinematic optimization of robotic manipulators". ASME Journal of Mechanical Design, 113(3), pp. 220-226.

[53] Cammarata, A., and Sinatra, R., 2014. "Elastodynamic optimization of a 3T1R parallel manipulator". Mechanism and Machine Theory, 73, pp. 184-196.

[54] Shabana, A., 2005. Dynamics of Multibody Systems. Cambridge University Press.

[55] Sigmund, O., and Maute, K., 2013. "Topology optimization approaches: A comparative review". Structural and Multidisciplinary Optimization, 48(6), pp. 1031-1055.

[56] Briot, S., and Goldsztejn, A., 2018. "Topology optimization of industrial robots: Application to a five-bar mechanism". Mechanism and Machine Theory, 120, pp. 30-56.

[57] Albers, A., Ottnad, J., and Häussler, W., 2007. "Methods for lightweight design of mechanical components in humanoid robots". In Proceedings of the 7th IEEERAS International Conference on Humanoid Robots, pp. 609-615.

[58] Albers, A., and Ottnad, J., 2008. "System based topology optimization as development tools for lightweight components in humanoid robots". In Proceedings of the 8th IEEE-RAS International Conference on Humanoid Robots, pp. 674-680.

[59] Kim, B., Yun, D., Lee, S., and Jang, G., 2016. “Topol- ogy optimization of industrial robots for system-level stiffness maximization by using part-level metamodels". Structural and Multidisciplinary Optimization, pp. 1-11.

[60] Bendsoe, M., and Sigmund, O., 1999. "Material interpolation schemes in topology optimization". Archive of Applied Mechanics, 69.

[61] Craig, R. R., and Bampton, M. C. C., 1968. "Coupling of substructures for dynamic analysis". AIAA Journal, 6(7).

[62] Norm EN 14358, 2016. Timber structures. calculation and verification of characteristic values.

[63] Kaci, L., 2018. “Conception et commande d'un robot industriel en bois". $\mathrm{PhD}$ thesis.

[64] Pagis, G., Bouton, N., Briot, S., and Martinet, P., 2015. "Enlarging parallel robot workspace through type-2 singularity crossing". Control Engineering Practice, 39, pp. 1-11.

[65] Pshenichnyj, B., 1994. The Linearization Method for Constrained Optimization. Computational Mathematics. Springer.

[66] Paccot, F., Andreff, N., and Martinet, P., 2009. "A review on the dynamic control of parallel kinematic machines: theory and experiments". The International Journal of Robotics Research, 28(3), pp. 395-416.

[67] Thais Colombo, F., de Carvalho Fontes, J., and da Silva, M., 2019. "A visual servoing strategy under limited frame rates for planar parallel kinematic machines". Journal of Intelligent $\mathcal{G}$ Robotic Systems, 96(1), pp. 95-107.

[68] Marchand, E., and Chaumette, F., 2001. A new formulation for non-linear camera calibration using virtual visual servoing. Tech. Rep. RR-4096, INRIA.

[69] Marchand, E., Spindler, F., and Chaumette, F., 2005. "ViSP for visual servoing: a generic software platformwith a wide class of robot control skills". pp. 4052.

[70] Visual Servoing Plateform (ViSP). Tutorial : How to boost your visual servo control law. Tech. rep., INRIA.

[71] Mansard, N., and Chaumette, F., 2007. "Task sequencing for high-level sensor-based control". IEEE Transactions on Robotics, 23(1), pp. 60-72.

[72] Uemura, M., and Kawamura, S., 2009. "Resonancebased motion control method for multijoint robot through combining stiffness adaptation and iterative learning control". In Proceedings of the IEEE International Conference on Robotics and Automation, pp. 1543-1548.

[73] Riazi, S., Bengtsson, K., Bischoff, R., Aurnhammer, A., Wigström, O., and Lennartson, B., 2016. "Energy and peak-power optimization of existing time-optimal robot trajectories". In Proceedings of the IEEE International Conference on Automation Science and Engineering.

[74] Vaughan, J., Yano, A., and Singhose, W. E., 2009. "Robust negative input shapers for vibration suppression". ASME Journal of Dynamic Systems, Measurement, and Control, 131(3). 
[75] Douat, L., Queinnec, I., Garcia, G., Michelin, M., and Pierrot, F., 2011. "Hinfiny control applied to the vibration minimization of the parallel robot Par2". In IEEE Multiconference on Systems and Control.

[76] Hryshaienko, O., 2018. RobEcolo: Life cycle analysis. Tech. rep., LS2N. 


\section{List of Tables}

\begin{tabular}{|c|c|}
\hline & Performance required for the wooden indus- \\
\hline & trial robot \\
\hline$\sqrt{2}$ & Optimal link lengths for the wooden five-bar \\
\hline & mechanism \\
\hline 3 & Optimal location of the camera \\
\hline 4 & Mean of the norm of the deformations in the \\
\hline & plane $\left(x_{0} O y_{0}\right)$ in $\mathrm{mm}$ for a horizontal load of \\
\hline & $20 \mathrm{~N}$. \\
\hline 5 & Mean of the vertical deformations in $\mathrm{mm}$ for \\
\hline & a vertical load of $20 \mathrm{~N}$. \\
\hline 6 & Repeatability measurements in microns for \\
\hline & the two types of controllers. \\
\hline 7 & Positionning accuracy measurements in mi- \\
\hline & crons for the two types of controllers. \\
\hline 8 & Number of robots with unacceptable defor- \\
\hline
\end{tabular}




\section{List of Figures}

\begin{tabular}{|c|c|}
\hline & $\mathrm{sm}$ \\
\hline 2 & ferent bodies for the five- \\
\hline & bar mechanism. \\
\hline & Observation of the end-effector of the \\
\hline & five-bar mechanism \\
\hline & Observation of a cylindrical leg of the \\
\hline & five-bar mechanism \\
\hline 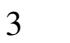 & Result of a one-pixel error on the intersec- \\
\hline & tion of the image boundary and the observed \\
\hline & bserved is in blue, its \\
\hline & pixel appearance is in purple, and the poten- \\
\hline & tial reconstruction errors are the lines in red. \\
\hline 4 & Largest regular dexterous workspace of the \\
\hline & optimized five-bar mechanism \\
\hline | & shed with finite elements. \\
\hline 6 & al links: initial design \\
\hline & domain \\
\hline 7 & al links: final results for \\
\hline & different values o \\
\hline 8 & oden five-bar mechanism. \\
\hline & Proximal Link: CAD Design \\
\hline & Final Prototype \\
\hline 9 & Final prototype of the RobEcolo robot. \\
\hline 10 & Interaction layout for Robecolo prototype. \\
\hline 11 & Tracker for detecting leg edges and end- \\
\hline & location. \\
\hline 12 & Robot links config \\
\hline & sis points. \\
\hline 13 & Benchmark for measuring the robot defor- \\
\hline & mations \\
\hline & ion to measure deforma- \\
\hline & tions along the vertical axis \\
\hline & easure deforma- \\
\hline & tions in the horizontal plane \\
\hline 14 & $g$ the robot accuracy: \\
\hline & ts $A, B, C, D$ \\
\hline & 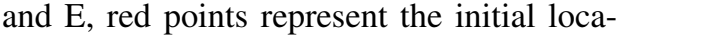 \\
\hline & \\
\hline
\end{tabular}

